MATHEMATICS OF COMPUTATION

Volume 79, Number 271, July 2010, Pages 1535-1564

S $0025-5718(10) 02326-4$

Article electronically published on March 23, 2010

\title{
WELL-POSEDNESS AND NUMERICAL ANALYSIS OF A ONE-DIMENSIONAL NON-LOCAL TRANSPORT EQUATION MODELLING DISLOCATIONS DYNAMICS
}

\author{
A. GHORBEL AND R. MONNEAU
}

\begin{abstract}
We consider a situation where dislocations are parallel lines moving in a single plane. For this simple geometry, dislocations dynamics is modeled by a one-dimensional non-local transport equation. We prove a result of existence and uniqueness for all time of the continuous viscosity solution for this equation. A finite difference scheme is proposed to approximate the continuous viscosity solution. We also prove an error estimate result between the continuous solution and the discrete solution, and we provide some simulations.
\end{abstract}

\section{INTRODUCTION}

1.1. Physical motivation. In this work, we are interested in the dislocations dynamics in a crystal material (see 18 for a physical description of dislocations). A perfect crystal, for small deformations, is well described by the equations of linear elasticity. The real crystals contain, in particular, some line defects called dislocations. The dislocations dynamics is one of the main explanations of the plastic deformation of metals. When we apply an exterior stress, these dislocations lines can move in a slip plane of the crystal. We consider here a simple geometry where the dislocations are parallel lines moving in a same plane $(x y)$. This plane is embedded in a three-dimensional elastic crystal. The particular geometry of this problem leads to the study of a one-dimensional model given by the following non-local transport equation modelling dislocations dynamics:

$$
\left\{\begin{array}{l}
\frac{\partial u}{\partial t}(x, t)=c[u](x, t) \frac{\partial u}{\partial x}(x, t) \quad \text { in } \mathbb{R} \times(0,+\infty), \\
u(x, 0)=u^{0}(x) \text { in } \mathbb{R}
\end{array}\right.
$$

where the solution $u$ is a scalar function, $\frac{\partial u}{\partial t}$ and $\frac{\partial u}{\partial x}$ are its time and space derivatives, respectively. Here the dislocations move with a non-local velocity $c[u]$ known

Received by the editor January 13, 2009.

2010 Mathematics Subject Classification. Primary 35F20, 35F25, 35K55, 49L25, 65N06, $65 \mathrm{~N} 12,74 \mathrm{~N} 05$.

Key words and phrases. Dislocations dynamics, Peach-Koehler force, transport equation, eikonal equation, Hamilton-Jacobi equation, non-local equation, continuous viscosity solutions, convergence of numerical scheme, finite difference scheme.

(C)2010 American Mathematical Society 
as the resolved Peach-Koehler force; see 20. It is given by

$$
\left\{\begin{array}{l}
c[u](x, t)=c^{\mathrm{ext}}(x)+c^{\mathrm{int}}[u](x, t), \\
c^{\mathrm{int}}[u](x, t)=\int_{\mathbb{R}} c^{0}\left(x-x^{\prime}\right)\left(E\left(u\left(x^{\prime}, t\right)\right)-P x^{\prime}\right) d x^{\prime},
\end{array}\right.
$$

where the function $E$ is the floor function defined by $E(v)=k$ if $k \leq v<k+1$, $k \in \mathbb{Z}$. The scalar function $u$ has no physical meaning but it is chosen such that the jumps of $E(u)$ correspond to the positions of dislocations (see Figure 1). The velocity $c[u]$ is the sum of two terms. We first assume the existence in the material of obstacles to the motion of dislocations. The term $c^{\text {ext }}$ represents the exterior stress created by these obstacles (such as precipitates in the material, other fixed dislocations, other defects, ...). We consider obstacles that are independent on time and periodic in space. Namely, we assume that the velocity satisfies

$$
c^{\text {ext }} \in W^{1, \infty}(\mathbb{R}) \quad \text { such that } \quad c^{\text {ext }}(x+1)=c^{\text {ext }}(x) \text { in } \mathbb{R} .
$$

The second term $c^{\mathrm{int}}[u]$ is a non-local term, given by a convolution with respect to the space variable, and represents the elastic interior stress created by all the dislocations in the material. This term $c^{\text {int }}[u]$ is obtained by the resolution of the equations of linear elasticity. For instance, in the model of Peierls-Nabarro (see [5]), we have in the case of edge dislocation (see [18])

$$
c^{0}(x)=\frac{-\mu b^{2}}{2 \pi(1-\nu)} \frac{x^{2}-\zeta^{2}}{\left(x^{2}+\zeta^{2}\right)^{2}} \quad \text { on } \mathbb{R},
$$

where $\nu=\frac{\lambda}{2(\lambda+\mu)}$ is the Poisson ratio and $\lambda$ and $\mu>0$ are the Lamé coefficients for isotropic elasticity. The Burgers vector $\vec{b}$ is equal to $b \vec{e}_{x}$, with $b>0$ and $\vec{e}_{x}$ the unit vector in the direction of $x$ of Figure 1. There is a physical parameter $\zeta \neq 0$ (depending on the material) which represents the size of the core of the dislocation.

1.2. Main results. In the rest of this paper, we use some adapted norms introduced in the following definition.

Definition 1.1 (Adapted norms). Let two functions $f \in L_{\text {loc }}^{1}(\mathbb{R})$ and $g \in L_{\text {loc }}^{\infty}(\mathbb{R})$. We define the quantities

$$
|f|_{L_{\text {unif }}^{1}(\mathbb{R})}=\sup _{x \in \mathbb{R}} \int_{I(x)}|f(y)| d y \quad \text { and } \quad|g|_{L_{\text {int }}^{\infty}(\mathbb{R})}=\int_{\mathbb{R}}|g|_{L^{\infty}(I(x))} d x,
$$

where $I(x)=\left(x-\frac{1}{2}, x+\frac{1}{2}\right)$. We denote respectively $L_{\text {unif }}^{1}(\mathbb{R})$ and $L_{\text {int }}^{\infty}(\mathbb{R})$ spaces that consist of functions for which these quantities are finite.

Remark 1.2. These spaces are motivated by the following fact. For $c^{0} \in L_{\text {int }}^{\infty}(\mathbb{R})$ and $f \in L_{\text {unif }}^{1}(\mathbb{R})$, we will show later that the convolution product $c^{0} \star f$ is well defined. This will be applied to define $c^{\text {int }}[u]$ with $f(x)=E(u(x, t))-P x$.

We denote $\operatorname{Lip}(\mathbb{R})$ the space of Lipschitz continuous functions on $\mathbb{R}$.

1.2.1. Existence and uniqueness of a continuous solution. We consider the following assumptions for the kernel $c^{0}$ :

$$
\left\{\begin{array}{l}
c^{0} \in W^{1,1}(\mathbb{R}) \cap L_{\text {int }}^{\infty}(\mathbb{R}), \\
c^{0}(x)=c^{0}(-x) \text { and } \int_{\mathbb{R}} c^{0}(x) d x=0 .
\end{array}\right.
$$




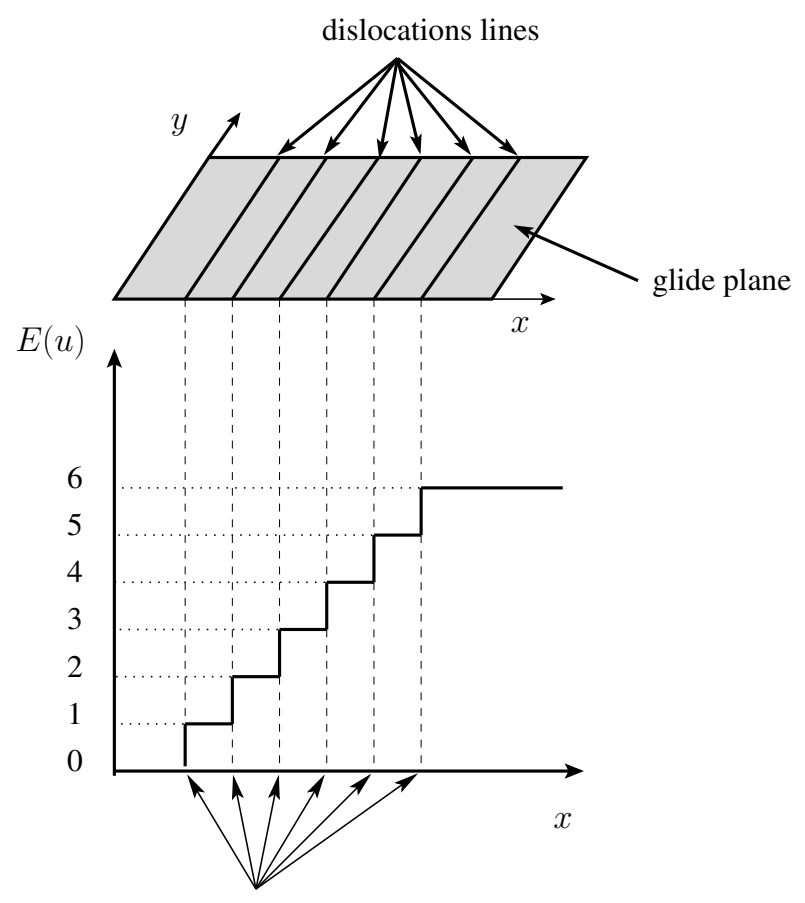

the jumps of $E(u)$ correspond

to the positions of dislocations

Figure 1. Representation of dislocations with the function $E(u)$

One can check easily that the kernel given in (44) satisfies (5). We consider the initial condition $u^{0} \in \operatorname{Lip}(\mathbb{R})$ such that for $x \in \mathbb{R}$

$$
u^{0}(x+1)=u^{0}(x)+P \quad \text { and } \quad 0<b_{0} \leq u_{x}^{0} \leq B_{0}<+\infty \quad \text { a.e. }
$$

with $b_{0}$ and $B_{0}$ some constants and $P \in \mathbb{N} \backslash\{0\}$. This condition means in particular that dislocations are periodically distributed. As mentioned above, in order to study the solutions of (1), we use the theory of continuous viscosity solutions (see [7, 10]). Our first main result is:

Theorem 1.3 (Long time existence and uniqueness of the solution). Under assumptions (3), (5), (6), there exists a unique continuous viscosity solution $u \in$ $W_{\mathrm{loc}}^{1, \infty}(\mathbb{R} \times[0,+\infty))$ of (1), (2) satisfying $u(x+1, t)=u(x, t)+P$.

In [5], a short time existence and uniqueness result is given for a two-dimensional problem for a single dislocation line. Because in the present work our problem is one-dimensional, we are able to get a refined result for the dynamics of several dislocations in interaction, namely the existence and uniqueness of a solution for all time.

Let us mention that under the more restrictive assumptions that the velocity $c[u]$ is nonnegative, the existence and uniqueness of a solution for all time is proved in [1, 9]. 
In the special case where the kernel $c^{0}$ is assumed nonnegative, some existence and uniqueness results for all time in any dimension are available in a "Slepčev formulation" (see [8, 14]).

The previous theorem will be proved in two steps. First, we will prove the result for short time (see for instance [17]) using a fixed point theorem. Second, we will repeat this short time result on a sequence of time intervals of lengths $T_{n}$ decreasing to zero, such that $\sum_{n \in \mathbb{N}} T_{n}=+\infty$.

Remark 1.4. Let us mention three remaining open problems.

- When the initial data $u^{0}$ is not monotone, the existence and uniqueness of the solution for all time is an open question.

- We do not know the behavior of the solution as $t \rightarrow+\infty$.

- If we replace $\frac{\partial u}{\partial x}$ in equation (11) by its absolute value, then we have a nonlocal Hamilton-Jacobi equation. Physically, the absolute value would allow us to consider the possible annihilation of two dislocations associated to opposed jumps of $E(u)$. The existence and uniqueness of a solution for all time is an open question in the general case. Nevertheless, in the whole paper we will only consider the case of solutions $u$ monotone in space which allows us to forget the absolute value.

1.2.2. Convergence of a numerical scheme. We build a finite difference scheme of order one in space and time

- by assuming that it is upwind,

- by approximating the non-local term $c^{0} \star E(u(\cdot, t))$ by a discrete convolution, and

- by using an explicit Euler scheme in time.

Given a mesh size $\Delta x, \Delta t$ and a lattice $I_{d}=\{(i \Delta x, n \Delta t) ; i \in \mathbb{Z}, n \in \mathbb{N}\},\left(x_{i}, t_{n}\right)$ denotes the node $(i \Delta x, n \Delta t)$ and $v^{n}=\left(v_{i}^{n}\right)_{i}$ the values of the numerical approximation of the continuous solution $u\left(x_{i}, t_{n}\right)$. We then consider the following numerical scheme:

$$
v_{i}^{0}=u^{0}\left(x_{i}\right), \quad v_{i}^{n+1}=v_{i}^{n}+\Delta t c_{i}\left(v^{n}\right) \times \begin{cases}D_{x}^{+} v_{i}^{n} & \text { if } c_{i}\left(v^{n}\right) \geq 0, \\ D_{x}^{-} v_{i}^{n} & \text { if } c_{i}\left(v^{n}\right)<0,\end{cases}
$$

with

$$
D_{x}^{+} v_{i}^{n}=\frac{v_{i+1}^{n}-v_{i}^{n}}{\Delta x}, \quad D_{x}^{-} v_{i}^{n}=\frac{v_{i}^{n}-v_{i-1}^{n}}{\Delta x},
$$

and $c_{i}\left(v^{n}\right)$ is defined below.

We choose $\Delta x=\frac{1}{K}, K \in \mathbb{N} \backslash\{0\}$ because of the 1-periodicity in space. We denote $c_{i}^{\text {ext }}=c^{\text {ext }}\left(x_{i}\right)$ which satisfies $c_{i+K}^{\text {ext }}=c_{i}^{\text {ext }}$. The discrete velocity is

$$
c_{i}\left(v^{n}\right)=c_{i}^{\mathrm{ext}}+c_{i}^{\mathrm{int}, \mathrm{n}} \text { with } c_{i}^{\mathrm{int}, \mathrm{n}}=\sum_{l \in \mathbb{Z}} c_{l}^{0} E\left(v_{i-l}^{n}\right) \Delta x,
$$

where

$$
c_{i}^{0}=\frac{1}{\Delta x} \int_{I_{i}} c^{0}(x) d x \quad \text { and } \quad I_{i}=\left[x_{i}-\frac{\Delta x}{2}, x_{i}+\frac{\Delta x}{2}\right] .
$$

We are interested in solutions $v^{n}$ satisfying $v_{i+K}^{n}=v_{i}^{n}+P$ for all $i \in \mathbb{Z}$. Then we can check that the discrete velocity satisfies $c_{i+K}\left(v^{n}\right)=c_{i}\left(v^{n}\right)$. 
Note that the global scheme $v^{n+1}=S\left(v^{n}\right)$ given by (7) is not monotone in general because the velocity $c_{i}\left(v^{n}\right)$ depends non-monotonically on the solution $v^{n}$ itself (here some $c_{i}^{0}$ are non-positive because we assumed that $\int_{\mathbb{R}} c^{0}(x) d x=0$ ).

We assume that the mesh satisfies the following CFL (Courant, Friedrichs, Lévy) condition

$$
\Delta t<\frac{\Delta x}{4\left(\left|c^{\text {ext }}\right|_{L^{\infty}(\mathbb{R})}+P\left|c^{0}\right|_{L^{1}(\mathbb{R})}\right)} .
$$

Our second main result is

Theorem 1.5 (Error estimate). Let $u$ be the continuous viscosity solution of problems (1), (2) under assumptions (3), (5), (6). Let $v$ be the discrete solution of the associated finite difference scheme (7)-(10). Assume that the time step $\Delta t$ satisfies

$$
\Delta t=\alpha \Delta x \quad \text { with } \quad 0<\alpha<\frac{1}{4\left(\left|c^{\text {ext }}\right|_{L^{\infty}(\mathbb{R})}+P\left|c^{0}\right|_{L^{1}(\mathbb{R})}\right)} .
$$

Then, there exists two constants $T_{1}, C>0$, depending on $\alpha,\left|c^{\text {ext }}\right|_{W^{1, \infty}(\mathbb{R})}, P$, $\left|c^{0}\right|_{W^{1,1}(\mathbb{R})},\left|c^{0}\right|_{L_{\text {int }}^{\infty}(\mathbb{R})}, B^{0}$ and $b^{0}$ in (6), such that

$$
\sup _{i \in \mathbb{Z}}\left|u(i \Delta x, n \Delta t)-v_{i}^{n}\right| \leq C|\Delta x|^{1 / 2} \quad \text { for all } n \leq \frac{T_{1}}{\Delta t} \quad \text { if } \quad \Delta x \leq \frac{T_{1}}{C} .
$$

The proof of this theorem is based on the ideas of Crandall and Lions [13] adapted to the case of non-local equations (see [2]).

Extensive simulations of dislocations dynamics are provided in [16.

1.3. Brief review of the literature. Let us recall that, in the 1980s, the notion of viscosity solution was first introduced by Crandall and Lions in [11] for first-order Hamilton-Jacobi equations. For an introduction to this notion, see in particular the books of Barles [7, and of Bardi and Capuzzo-Dolcetta [6].

Recently, Alvarez, Hoch, Le Bouar and Monneau [4, 5] used this theory for the resolution of a non-local Hamilton-Jacobi equation modelling dislocation dynamics. They proved results of short time existence and uniqueness of a discontinuous viscosity solution. Their results are mainly valid for dislocations with the shape of graphs and loops, and they used the level set approach, which was introduced by Osher and Sethian [19]. As already mentioned, in the situation where the non-local velocity is non-negative, Barles and Ley [9] proved that the existence and uniqueness is valid for any time interval for a level set formulation. Still in the case of non-negative velocity, an approach for discontinuous viscosity solution was developed by Alvarez, Cardaliaguet and Monneau 11. Let us mention, for dislocations dynamics with mean curvature terms, Forcadel in [15] proved a short time existence and uniqueness result.

A numerical analysis was done by Crandall and Lions [13, for approximations of solutions of Hamilton-Jacobi equations. Convergence of a first-order scheme for an abstract non-local eikonal equation was proved by Alvarez, Carlini, Monneau and Rouy 2. They also applied this convergence result for the numerical analysis of a non-local Hamilton-Jacobi equation in [3] describing the dynamics of a single dislocation in two dimensions. 
1.3.1. Organization of the paper. In Section 2 we give some properties of the solution of an auxiliary local equation, i.e. an eikonal equation where the velocity is assumed to be a given function independent on the solution. In Section 3, we give some properties of the non-local velocity. The existence and uniqueness result of a continuous solution, i.e. Theorem 1.3. is then proved in Section 4 . We give preliminary results for the discrete local problem in Section 5 and for the discrete non-local velocity in Section 6. Theorem [1.5] about the error estimate is proved in Section 7 . Finally, in Section 8 we give some simulations.

\section{Preliminary Results for the eikonal EQuation WITH PRESCRIBED VELOCITY}

In this section, we start by recalling the notion of viscosity solution of an eikonal equation. We then give some properties of the solution of a such equation.

Let $T>0$. Consider the following problem:

$$
\begin{cases}\frac{\partial u}{\partial t}(x, t)=c(x, t)\left|\frac{\partial u}{\partial x}(x, t)\right| & \text { in } \mathbb{R} \times(0, T), \\ u(x, 0)=u_{0}(x) & \text { on } \mathbb{R} .\end{cases}
$$

We make the following assumptions:

a) the velocity $c: \mathbb{R} \times(0, T) \longrightarrow \mathbb{R}$ is bounded, Lipschitz continuous in space and in time,

b) the initial data $u_{0} \in \operatorname{Lip}(\mathbb{R})$.

We recall the notions of viscosity subsolutions, supersolutions and solutions for (13) (see [10]). We denote

$\mathrm{USC}(\mathbb{R} \times[0, T))=\{u: \mathbb{R} \times[0, T) \longrightarrow \mathbb{R}$, locally bounded, upper semicontinuous $\}$

and

$\operatorname{LSC}(\mathbb{R} \times[0, T))=\{u: \mathbb{R} \times[0, T) \longrightarrow \mathbb{R}$, locally bounded, lower semicontinuous $\}$.

We then define

Definition 2.1 (Viscosity subsolution, supersolution and solution).

1) A function $u \in U S C(\mathbb{R} \times[0, T))$ is a viscosity subsolution of (13) if the following properties hold:

i) $u(x, 0) \leq u_{0}(x)$ in $\mathbb{R}$,

ii) for every $\left(x_{0}, t_{0}\right) \in \mathbb{R} \times(0, T)$ and for every test function $\varphi \in$ $C^{1}(\mathbb{R} \times(0, T))$ such that $u-\varphi$ has a local maximum at $\left(x_{0}, t_{0}\right)$, we have

$$
\frac{\partial \varphi}{\partial t}\left(x_{0}, t_{0}\right) \leq c\left(x_{0}, t_{0}\right)\left|\frac{\partial \varphi}{\partial x}\left(x_{0}, t_{0}\right)\right| .
$$

2) A function $u \in L S C(\mathbb{R} \times[0, T))$ is a viscosity supersolution of (13) if the following properties hold:

i) $u(x, 0) \geq u_{0}(x)$ in $\mathbb{R}$,

ii) for every $\left(x_{0}, t_{0}\right) \in \mathbb{R} \times(0, T)$ and for every test function $\phi \in$ $C^{1}(\mathbb{R} \times(0, T))$ such that $u-\phi$ has a local minimum at $\left(x_{0}, t_{0}\right)$, we have

$$
\frac{\partial \phi}{\partial t}\left(x_{0}, t_{0}\right) \geq c\left(x_{0}, t_{0}\right)\left|\frac{\partial \phi}{\partial x}\left(x_{0}, t_{0}\right)\right| .
$$


3) A function $u \in C(\mathbb{R} \times[0, T))$ is a continuous viscosity solution of (13) if it is both a viscosity subsolution and a viscosity supersolution of (13).

We have the following a priori estimates for the solution of the eikonal equation. These estimates are may be quite classical, and part of them is already proved in [5, but we give a proof for sake of completeness.

Proposition 2.2 (a priori estimates for the solution of the eikonal equation). Assume that $c \in W^{1, \infty}(\mathbb{R} \times[0, T])$ and $u_{0} \in \operatorname{Lip}(\mathbb{R})$ such that $\left|\left(u_{0}\right)_{x}\right| \leq B_{0}$ a.e. and $\left(u_{0}\right)_{x} \geq b_{0}$ a.e. for some $B_{0}>b_{0}>0$. Then, there exists a unique continuous viscosity solution $u$ on $\mathbb{R} \times[0, T)$ of problem (13). Moreover, $u \in \operatorname{Lip}(\mathbb{R} \times[0, T)$ ). With $L_{c}:=L_{c}(t)=\left|c_{x}(\cdot, t)\right|_{L^{\infty}(\mathbb{R})}, B(t)=B_{0} e^{L_{c} t}$ and $b(t)=b_{0} e^{-L_{c} t}$, we have the following estimates:

i) for every $0 \leq t<T$,

$$
\left|u_{x}(x, t)\right| \leq B(t) \quad \text { a.e. }
$$

and

$$
u_{x}(x, t) \geq b(t) \quad \text { a.e. }
$$

ii) Moreover

$$
\left|u_{t}(x, t)\right| \leq|c|_{L^{\infty}(\mathbb{R} \times(0, T))} B(t) \quad \text { a.e. }
$$

Proof of Proposition 2.2. We refer to [7, Theorem 2.8, page 38] for the proof of existence and uniqueness of a solution $u$. We introduce the double variables $(x, y) \in$ $\mathbb{R}^{2}$ and set the half-plane $\Omega=\{x \geq y\}$. Consider the following problem:

$$
\begin{cases}w_{t}(x, y, t)=c(x, t)\left|w_{x}(x, y, t)\right|-c(y, t)\left|w_{y}(x, y, t)\right| & \text { in } \Omega \times(0, T), \\ w(x, y, 0)=u(x, 0)-u(y, 0) & \text { in } \Omega, \\ w(x, x, t)=0 & \text { on } \partial \Omega \times(0, T) .\end{cases}
$$

Then, $w(x, y, t)=u(x, t)-u(y, t)$ is a continuous viscosity solution of problem (14) (we refer to [12, Lemma 2, page 357] for a proof).

Let $\Phi(x, y, t)=B(t)(x-y)$. Then, we have

Claim 1. $\Phi$ is a (viscosity) supersolution of problem (14).

As a matter of fact, since $\Phi$ is smooth, $\Phi$ is a classical supersolution of problem (14). Indeed, on the one hand, we have

$$
\begin{aligned}
& w(x, y, 0)=u(x, 0)-u(y, 0) \leq B_{0}(x-y)=\Phi(x, y, 0) \quad \text { and } \\
& w(x, x, t)=0=\Phi(x, x, t) .
\end{aligned}
$$

On the other hand, we have

$$
\begin{aligned}
& \Phi_{t}-c(x, t)\left|\Phi_{x}\right|+c(y, t)\left|\Phi_{y}\right| \\
= & L_{c} B_{0} e^{L_{c} t}(x-y)-c(x, t) B_{0} e^{L_{c} t}+c(y, t) B_{0} e^{L_{c} t} \\
= & B_{0} e^{L_{c} t}\left(-c(x, t)+c(y, t)+L_{c}(x-y)\right) .
\end{aligned}
$$

Moreover,

$|c(x, t)-c(y, t)| \leq L_{c}|x-y| \quad$ and $\quad x \geq y \quad$ implies $\quad c(x, t)-c(y, t) \leq L_{c}(x-y)$.

We then obtain

This proves Claim 1 .

$$
\Phi_{t}-c(x, t)\left|\Phi_{x}\right|+c(y, t)\left|\Phi_{y}\right| \geq 0 .
$$

Let $\varphi(x, y, t)=b_{0} e^{-L_{c} t}(x-y)$. Then we have 
Claim 2. $\varphi$ is a (viscosity) subsolution of problem (14).

The proof is similar to the proof of Claim 1, and we skip it.

By the comparison principle (see [7, Theorem 2.10, page 47]):

a)

$$
\begin{gathered}
w(x, y, t) \leq \Phi(x, y, t) \quad \text { i.e. } \\
u(x, t)-u(y, t) \leq B(t)(x-y) \quad \text { for }(x, y, t) \in \Omega \times(0, T),
\end{gathered}
$$

b) and

$$
\begin{gathered}
\varphi(x, y, t) \leq w(x, y, t) \quad \text { i.e. } \\
b(t)(x-y) \leq u(x, t)-u(y, t) \quad \text { for }(x, y, t) \in \Omega \times(0, T) .
\end{gathered}
$$

We deduce that

$$
0 \leq b(t)(x-y) \leq u(x, t)-u(y, t) \leq B(t)(x-y) \leq B(t)|x-y|
$$

for all $(x, y, t) \in \Omega \times(0, T)$. Passing to the limit in (17), by Rademacher's Theorem [7, we get

$$
0 \leq b(t) \leq u_{x}(x, t) \leq B(t) \text { for a.e. }(x, t) \in \Omega \times[0, T) .
$$

We now prove the Lipschitz in time estimate. Let $\left(x_{0}, t_{0}\right) \in \mathbb{R} \times(0, T)$ and $\varphi \in$ $C^{1}(\mathbb{R} \times(0, T))$ such that $u-\varphi$ has a local maximum at $\left(x_{0}, t_{0}\right)$. We show that $\varphi_{t} \leq$ $|c|_{L^{\infty}(\mathbb{R} \times(0, T))} B\left(t_{0}\right)$. From $\frac{\varphi\left(x_{0}, t_{0}\right)-\varphi\left(x, t_{0}\right)}{\left|x-x_{0}\right|} \leq \frac{u\left(x_{0}, t_{0}\right)-u\left(x, t_{0}\right)}{\left|x-x_{0}\right|}$ and (17) we obtain

$$
\varphi_{x}\left(x_{0}, t_{0}\right) \leq B\left(t_{0}\right)
$$

and then

$\varphi_{t}\left(x_{0}, t_{0}\right) \leq c\left(x_{0}, t_{0}\right)\left|\varphi_{x}\left(x_{0}, t_{0}\right)\right| \leq|c|_{L^{\infty}(\mathbb{R} \times(0, T))}\left|\varphi_{x}\left(x_{0}, t_{0}\right)\right| \leq|c|_{L^{\infty}(\mathbb{R} \times(0, T))} B\left(t_{0}\right)$.

Let $\Phi \in C^{1}(\mathbb{R} \times(0, T))$ such that $u-\varphi$ has a local maximum at $\left(x_{0}, t_{0}\right) \in \mathbb{R} \times(0, T)$. Similarly, we check easily that $\Phi_{t} \geq-|c|_{L^{\infty}(\mathbb{R} \times(0, T))} B\left(t_{0}\right)$. Therefore, we have

$$
\varphi_{t} \leq|c|_{L^{\infty}(\mathbb{R} \times(0, T))} B\left(t_{0}\right) \quad \text { and } \quad \Phi_{t} \geq-|c|_{L^{\infty}(\mathbb{R} \times(0, T))} B\left(t_{0}\right) .
$$

We conclude that

$$
\left|u_{t}\right| \leq|c|_{L^{\infty}(\mathbb{R} \times(0, T))} B\left(t_{0}\right) \text { in the viscosity sense. }
$$

We now give a stability result.

Proposition 2.3 (Stability of the solution by perturbation of the velocity). Let $v^{i}, i=1,2$, be a viscosity solution of the problem

$$
\left\{\begin{array}{rlrl}
v_{t}^{i}(x, t) & =c^{i}(x, t)\left|v_{x}^{i}(x, t)\right| & \text { in } & \mathbb{R} \times[0, T), \\
v^{i}(x, 0) & =u_{0}(x) & \text { on } \mathbb{R}
\end{array}\right.
$$


where $c^{i} \in W^{1, \infty}(\mathbb{R} \times[0, T])$ and $u_{0} \in \operatorname{Lip}(\mathbb{R})$. Then,

$$
\begin{aligned}
& \left|v^{1}-v^{2}\right|_{L^{\infty}(\mathbb{R} \times[0, T])} \\
& \quad \leq \int_{0}^{T} d s\left|c^{2}(\cdot, s)-c^{1}(\cdot, s)\right|_{L^{\infty}(\mathbb{R})} \max \left(\left|v_{x}^{1}(\cdot, s)\right|_{L^{\infty}(\mathbb{R})},\left|v_{x}^{2}(\cdot, s)\right|_{L^{\infty}(\mathbb{R})}\right) .
\end{aligned}
$$

This result has been proved in [5]. For sake of completeness we give it here.

Proof of Proposition 2.3. We denote

$$
\bar{v}^{2}(x, t)=v^{2}(x, t)-\int_{0}^{t}\left|c^{2}(\cdot, s)-c^{1}(\cdot, s)\right|_{L^{\infty}(\mathbb{R})}\left|v_{x}^{2}(\cdot, s)\right|_{L^{\infty}(\mathbb{R})} d s .
$$

We want to prove that $\bar{v}^{2}$ is a viscosity subsolution of the equation satisfied by $v^{1}$. We denote $I(v)=v_{t}-c^{1}(x, t)\left|v_{x}\right|$. Formally, $I\left(v^{1}\right)=0$ and $I\left(v^{2}\right)=v_{t}^{2}-$ $c^{1}(x, t)\left|v_{x}^{2}\right|=\left(c^{2}(x, t)-c^{1}(x, t)\right)\left|v_{x}^{2}\right|$. We show that

$$
I\left(v^{2}\right) \leq\left|c^{2}(\cdot, t)-c^{1}(\cdot, t)\right|_{L^{\infty}(\mathbb{R})}\left|v_{x}^{2}(\cdot, t)\right|_{L^{\infty}(\mathbb{R})}
$$

in the viscosity sense. Indeed, let $\varphi \in C^{1}(\mathbb{R} \times(0, T))$ such that $v^{2}-\varphi$ has a local maximum at $\left(x_{0}, t_{0}\right) \in \mathbb{R} \times(0, T)$. Then,

$$
I(\varphi)=\varphi_{t}-c^{1}(x, t)\left|\varphi_{x}\right| \leq\left(c^{2}(x, t)-c^{1}(x, t)\right)\left|\varphi_{x}\right| \leq\left|c^{2}(\cdot, t)-c^{1}(\cdot, t)\right|_{L^{\infty}(\mathbb{R})}\left|\varphi_{x}\right| .
$$

Similarly, setting $\Phi \in C^{1}(\mathbb{R} \times(0, T))$ such that $v^{2}-\Phi$ has a local minimum at $\left(x_{0}, t_{0}\right) \in \mathbb{R} \times(0, T)$, we have

$$
I(\Phi) \geq-\left|c^{2}(\cdot, t)-c^{1}(\cdot, t)\right|_{L^{\infty}(\mathbb{R})}\left|\Phi_{x}\right| .
$$

Moreover, at $t=0$, we have $\bar{v}^{2}=v^{2}=u_{0}=v^{1}$. Hence, we deduce that $\bar{v}^{2}$ is a subsolution of the equation satisfied by $v^{1}$. Then, by the comparison principle [7, for all $t \in[0, T]$, we have $\bar{v}^{2} \leq v^{1}$, i.e.

$$
v^{2}(x, t)-v^{1}(x, t) \leq \int_{0}^{t}\left|c^{2}(\cdot, s)-c^{1}(\cdot, s)\right|_{L^{\infty}(\mathbb{R})}\left|v_{x}^{2}(\cdot, s)\right|_{L^{\infty}(\mathbb{R})} d s .
$$

Similarly, we prove the inequality $\bar{v}^{1} \geq v^{2}$ which leads to

$$
v^{2}(x, t)-v^{1}(x, t) \geq-\int_{0}^{t}\left|c^{2}(\cdot, s)-c^{1}(\cdot, s)\right|_{L^{\infty}(\mathbb{R})}\left|v_{x}^{1}(\cdot, s)\right|_{L^{\infty}(\mathbb{R})} d s .
$$

We conclude that

$$
\begin{aligned}
& \left|v^{2}-v^{1}\right|_{L^{\infty}(\mathbb{R} \times[0, T])} \\
& \quad \leq \int_{0}^{T}\left|c^{2}(\cdot, s)-c^{1}(\cdot, s)\right|_{L^{\infty}(\mathbb{R})} \max \left(\left|v_{x}^{1}(\cdot, s)\right|_{L^{\infty}(\mathbb{R})},\left|v_{x}^{2}(\cdot, s)\right|_{L^{\infty}(\mathbb{R})}\right) d s .
\end{aligned}
$$

\section{Properties of the NON-LOCAL Velocity}

The goal of this section is to prove the following estimate, which will be used in Section 4. 
Proposition 3.1 (Estimate on the difference of integer parts in the continuous case). Let $\rho^{1} \in C(\mathbb{R})$ such that:

i)

$$
\rho^{1}(x+1)=\rho^{1}(x)+P, \quad \text { where } P \in \mathbb{N} \backslash\{0\} ;
$$

ii) there exist constants $B \geq b>0$ such that $b \leq \rho_{x}^{1} \leq B$ in the distribution sense.

Let $\rho^{2} \in L_{\mathrm{loc}}^{\infty}(\mathbb{R})$ satisfying (20). Then,

$$
\left|E\left(\rho^{2}\right)-E\left(\rho^{1}\right)\right|_{L_{\text {unif }}^{1}(\mathbb{R})} \leq \frac{2}{b}\left(P+\left|\rho^{2}-\rho^{1}\right|_{L^{\infty}(\mathbb{R})}\right)\left|\rho^{2}-\rho^{1}\right|_{L^{\infty}(\mathbb{R})} .
$$

Remark 3.2. Note that if $\left|\rho^{2}-\rho^{1}\right|_{L^{\infty}(\mathbb{R})} \leq 1 \leq P$, then the previous estimate (21) becomes

$$
\left|E\left(\rho^{2}\right)-E\left(\rho^{1}\right)\right|_{L_{\text {unif }}^{1}(\mathbb{R})} \leq \frac{4 P}{b}\left|\rho^{2}-\rho^{1}\right|_{L^{\infty}(\mathbb{R})} .
$$

We will use this estimate later.

This result is the generalization of Lemma 4.2 in 3 to the case of several dislocations where the characteristic function $\rho^{i}>0$ is replaced with the floor part $E\left(\rho^{i}\right)$.

To do the proof of Proposition 3.1 we need to introduce the following notation.

We denote $\Lambda=\left|\rho^{2}-\rho^{1}\right|_{L^{\infty}(\mathbb{R})}$, and we assume that $\Lambda \in(0,+\infty)$ (other cases are trivial). For $k \in \mathbb{Z}$, we denote, for $i=1,2$,

$$
E_{k}^{i}=\left\{x \in \mathbb{R}, \rho^{i}(x)<k+1\right\} .
$$

First, we remark that since $\rho_{x}^{1} \geq b>0$ and $\rho^{1}$ is continuous, there exists a unique $a_{k} \in \mathbb{R}$ such that $\rho^{1}\left(a_{k}\right)=k+1$ and we have $E_{k}^{1}=\left(-\infty, a_{k}\right)$. We will use the following lemma for the proof of Proposition 3.1.

Lemma 3.3 (Estimate of the distance between the sets $E_{k}^{1}$ and $E_{k}^{2}$ ). With the notations introduced above and the assumptions of Proposition 3.1, we have

$$
E_{k}^{1}-\frac{\Lambda}{b} \subset E_{k}^{2} \subset E_{k}^{1}+\frac{\Lambda}{b} .
$$

Proof of Lemma 3.3. The main idea in this proof is to use the minoration of the gradient of the function $\rho^{1}$, i.e. $\rho_{x}^{1} \geq b>0$.

Let us first check that $E_{k}^{1}-\frac{\Lambda}{b} \subset E_{k}^{2}$. Let $x \in E_{k}^{1}-\frac{\Lambda}{b}$. Then, $x<a_{k}-\frac{\Lambda}{b}$, i.e. $\Lambda<b\left(a_{k}-x\right)$. Since $\rho_{x}^{1} \geq b>0$ and $a_{k}-x>0$, we have

$$
\rho^{1}\left(a_{k}\right)-\rho^{1}(x) \geq b\left(a_{k}-x\right),
$$

which implies (by definition of $\Lambda$ )

$$
k+1=\rho^{1}\left(a_{k}\right)>\rho^{1}(x)+\Lambda \geq \rho^{2}(x)
$$

and therefore

$$
k+1>\rho^{2}(x) .
$$

Thus, $x \in E_{k}^{2}$ for every $x \in E_{k}^{1}-\frac{\Lambda}{b}$ and therefore $E_{k}^{1}-\frac{\Lambda}{b} \subset E_{k}^{2}$ (see Figure 2). The second inclusion can be proved similarly. 


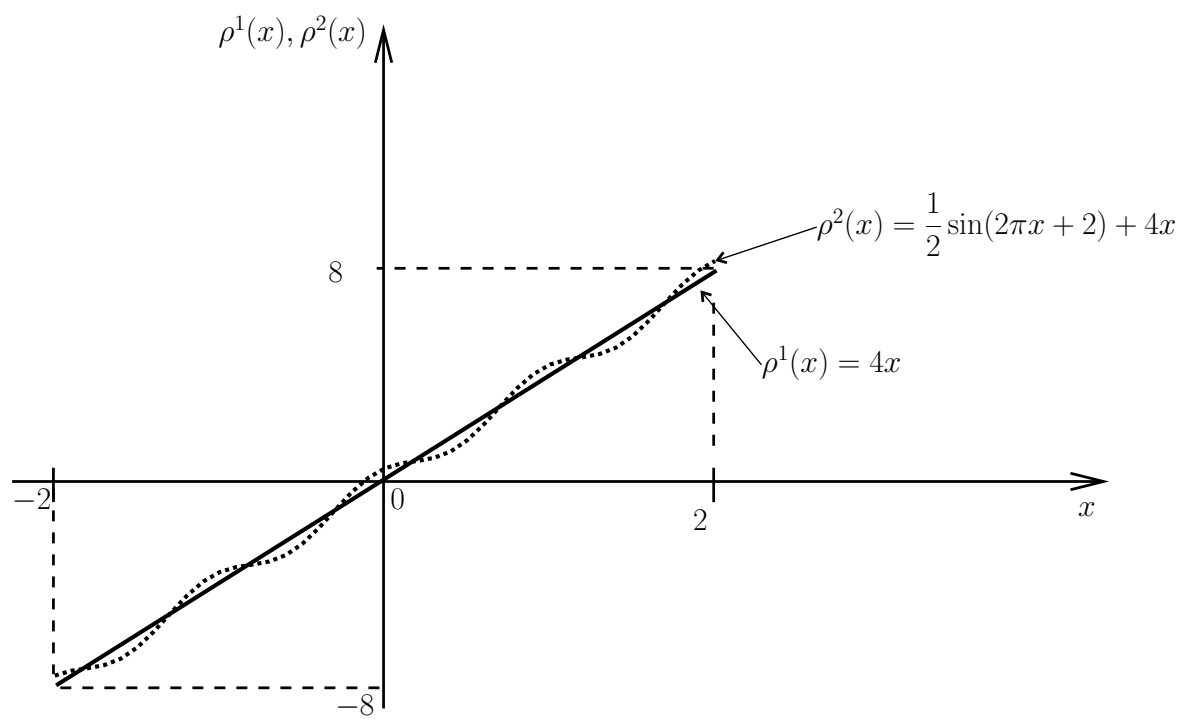

Figure 2. Example of functions $\rho^{1}$ and $\rho^{2}$ satisfying (20)

Proof of Proposition 3.1. The main idea in this proof is to bound the function $\left|E\left(\rho^{2}(x)\right)-E\left(\rho^{1}(x)\right)\right|$ by the characteristic functions of the sets $E_{k}^{2} \triangle E_{k}^{1}$. We then bound its $L_{\text {unif }}^{1}$-norm.

From the definition of $E_{k}^{i}$, for $i=1,2$, we remark that $E_{k-1}^{i} \subset E_{k}^{i}$ then $E\left(\rho^{i}(x)\right)=k$ if $x \in E_{k}^{i} \backslash E_{k-1}^{i}$, for $i=1,2$. We can write

$$
E\left(\rho^{i}(x)\right)=\sum_{k \in \mathbb{N}} 1_{\left(E_{k}^{i}\right)^{c}}(x)-\sum_{k \in \mathbb{Z} \backslash \mathbb{N}} 1_{E_{k}^{i}}(x) .
$$

Then,

$$
E\left(\rho^{2}(x)\right)-E\left(\rho^{1}(x)\right)=\sum_{k \in \mathbb{N}}\left(1_{\left(E_{k}^{2}\right)^{c}}(x)-1_{\left(E_{k}^{1}\right)^{c}}(x)\right)-\sum_{k \in \mathbb{Z} \backslash \mathbb{N}}\left(1_{E_{k}^{2}}(x)-1_{E_{k}^{1}}(x)\right) .
$$

Therefore,

$$
\begin{gathered}
\left|E\left(\rho^{2}(x)\right)-E\left(\rho^{1}(x)\right)\right| \leq \sum_{k \in \mathbb{N}} 1_{\left(E_{k}^{2}\right)^{c} \Delta\left(E_{k}^{1}\right)^{c}}(x)+\sum_{k \in \mathbb{Z} \backslash \mathbb{N}} 1_{E_{k}^{2} \Delta E_{k}^{1}}(x) \quad \text { a.e., } \\
\left|E\left(\rho^{2}(x)\right)-E\left(\rho^{1}(x)\right)\right| \leq \sum_{k \in \mathbb{Z}} 1_{E_{k}^{2} \Delta E_{k}^{1}}(x) \quad \text { a.e., }
\end{gathered}
$$

where $E_{k}^{2} \Delta E_{k}^{1}=\left(E_{k}^{2} \backslash E_{k}^{1}\right) \cup\left(E_{k}^{1} \backslash E_{k}^{2}\right)=\left(E_{k}^{2}\right)^{c} \Delta\left(E_{k}^{1}\right)^{c}$. By Lemma 3.3. $\left|E_{k}^{2} \triangle E_{k}^{1}\right| \leq \frac{2 \Lambda}{b}$. Then, we estimate for every $x \in \mathbb{R}$ with $I(x)=\left(x-\frac{1}{2}, x+\frac{1}{2}\right)$.

$$
\left|E\left(\rho^{2}\right)-E\left(\rho^{1}\right)\right|_{L^{1}(I(x))} \leq \sum_{k \in \mathbb{Z}}\left|I(x) \cap\left(E_{k}^{2} \Delta E_{k}^{1}\right)\right| \leq N^{\prime} \frac{2 \Lambda}{b},
$$


where

$$
N^{\prime}=\operatorname{Card}\left\{k \in \mathbb{Z},\left|I(x) \cap\left(E_{k}^{2} \Delta E_{k}^{1}\right)\right| \neq 0\right\} .
$$

Let us assume that there exists $k \in \mathbb{Z}$ such that $\left|I(x) \cap\left(E_{k}^{2} \triangle E_{k}^{1}\right)\right| \neq 0$. Then there exists $x^{1} \in I(x)$ such either $x^{1} \in\left(E_{k}^{1} \backslash E_{k}^{2}\right)$ or $x^{1} \in\left(E_{k}^{2} \backslash E_{k}^{1}\right)$. In the second case $\left(x^{1} \in\left(E_{k}^{2} \backslash E_{k}^{1}\right)\right)$, one can check easily in Figure 2 that the number of $k$ is less than $P+\left|\rho^{2}-\rho^{1}\right|_{L^{\infty}(\mathbb{R})}$. Therefore

$$
N^{\prime} \leq P+\left|\rho^{2}-\rho^{1}\right|_{L^{\infty}(\mathbb{R})} .
$$

Taking the supremum on $x \in \mathbb{R}$, we get

$$
\left|E\left(\rho^{2}\right)-E\left(\rho^{1}\right)\right|_{L_{\mathrm{unif}}^{1}(\mathbb{R})} \leq \frac{2}{b}\left(P+\left|\rho^{2}-\rho^{1}\right|_{L^{\infty}(\mathbb{R})}\right)\left|\rho^{2}-\rho^{1}\right|_{L^{\infty}(\mathbb{R})} .
$$

We recall the following result (we refer to [5] for a proof).

Lemma 3.4 (Norm of the product of convolution). For every $f \in L_{\text {unif }}^{1}(\mathbb{R})$ and $g \in L_{\mathrm{int}}^{\infty}(\mathbb{R})$, the convolution product $f \star g$ is bounded and satisfies

$$
|f \star g|_{L^{\infty}(\mathbb{R})} \leq|f|_{L_{\text {unif }}^{1}(\mathbb{R})}|g|_{L_{\text {int }}^{\infty}(\mathbb{R})} .
$$

We now present some properties of the non-local velocity.

Lemma 3.5 (Properties of the non-local velocity). Recall that $c^{\text {int }}[u](x, t)=$ $c^{0} \star(E(u(\cdot, t))-P \cdot)(x)$ is a convolution on $\mathbb{R}$. We assume that $c^{0}$ is a kernel in $W^{1,1}(\mathbb{R}) \cap L_{\mathrm{int}}^{\infty}(\mathbb{R})$ satisfying $\int_{\mathbb{R}} c^{0}(x) d x=0$. Then we have the following properties:

(1) The convolution $c^{\text {int }}$ is well defined if $u_{x} \geq 0$ a.e. and if $u(x+1, t)=$ $u(x, t)+P$ with $P \in \mathbb{N} \backslash\{0\}$;

(2) The function $c^{\text {int }}$, moreover, is 1-periodic in space, i.e. $c^{\mathrm{int}}[u](x+1, t)=$ $c^{\text {int }}[u](x, t)$. We also have $c^{\text {int }} \in L^{\infty}\left((0, T), W^{1, \infty}(\mathbb{R})\right)$, i.e. more precisely

$\left|c^{\mathrm{int}}[u](\cdot, t)\right|_{L^{\infty}(\mathbb{R})} \leq P\left|c^{0}\right|_{L^{1}(\mathbb{R})} \quad$ and $\quad\left|c_{x}^{\mathrm{int}}[u](\cdot, t)\right|_{L^{\infty}(\mathbb{R})} \leq P\left|\left(c^{0}\right)_{x}\right|_{L^{1}(\mathbb{R})} ;$

(3) If there exists $A>0$ such that $|u(x, t)-u(x, s)| \leq A|t-s|$ for a.e. $t, s \in$ $(0, T)$ and $u_{x} \geq b$ a.e., then $c^{\text {int }}$ is Lipschitz continuous in time with Lipschitz constant $\frac{4 A P}{b}\left|c^{0}\right|_{L_{\mathrm{int}}^{\infty}(\mathbb{R})}$, i.e.

$$
\left|c^{\mathrm{int}}(x, t)-c^{\mathrm{int}}(x, s)\right| \leq \frac{4 A P}{b}\left|c^{0}\right|_{L_{\mathrm{int}}^{\infty}(\mathbb{R})}|t-s| .
$$

Proof of Lemma 3.5.

(1) From $u(x+1, t)=u(x, t)+P$, we deduce that $E(u(x+1, t))=E(u(x, t))+$ $P$ and $E(u(x+1, t))-P(x+1)=E(u(x, t))-P x$. Since $\int_{\mathbb{R}} c^{0}(x) d x=0$, $\left(c^{0} \star P\right)(x)=0$, then $c^{0} \star(E(u(\cdot, t))-P \cdot)(x+1)=c^{0} \star(E(u(\cdot, t))-P \cdot)(x)$. Point 1 , therefore, is proved.

(2) Since $u(x+1, t)=u(x, t)+P$ and $u_{x} \geq 0$ for a.e. $(x, t) \in \mathbb{R} \times[0,+\infty)$, we have

$u(0, t) \leq u(x, t) \leq u(1, t)=u(0, t)+P \quad$ for all $(x, t) \in[0,1[\times[0,+\infty)$. 
Passing to the floor part, for $x \in[0,1[$ we obtain

$$
E(u(0, t)) \leq E(u(x, t)) \leq E(u(0, t))+P .
$$

Then

$$
-P \leq 0 \leq E(u(x, t))-E(u(0, t)) \leq P,
$$

and then $|E(u(x, t))-E(u(0, t))| \leq P$ for every $x \in[0,1[$ and every $t \geq 0$. We remark first that $c^{0} \star E(u(\cdot, t))(x)=c^{0} \star(E(u(\cdot, t))-E(u(0, t)))(x)$ because $\int_{\mathbb{R}} c^{0}(x) d x=0$. Then

$$
\begin{aligned}
& c^{0} \star(E(u(\cdot, t))-P \cdot)(x)=\int_{\mathbb{R}} d y c^{0}(x-y)(E(u(y, t))-P y-E(u(0, t))) \\
& =\sum_{k \in \mathbb{Z}} \int_{k}^{k+1} d y c^{0}(x-y)(E(u(y, t))-P y-E(u(0, t))) \\
& =\sum_{k \in \mathbb{Z}} \int_{0}^{1} d y c^{0}(x-y-k)(E(u(y+k, t))-P(y+k)-E(u(0, t))) \\
& =\sum_{k \in \mathbb{Z}} \int_{0}^{1} d y c^{0}(x-y-k)(E(u(y, t))-P y-E(u(0, t))) .
\end{aligned}
$$

Since $E(u(y, t))-E(u(0, t)) \leq P$ for $y \in[0,1[$, we deduce

$$
\begin{aligned}
c^{0} \star(E(u(\cdot, t))-P \cdot)(x) & \leq \sum_{k \in \mathbb{Z}} \int_{0}^{1} d y P(1-y) c^{0}(x-y-k) \\
& \leq P \int_{\mathbb{R}} d y c^{0}(x-y)=P \int_{\mathbb{R}} d y^{\prime} c^{0}\left(y^{\prime}\right) \\
& \leq P\left|c^{0}\right|_{L^{1}(\mathbb{R})},
\end{aligned}
$$

where for the last inequality we have used that $c^{0}(-x)=c^{0}(x)$ for all $x \in \mathbb{R}$ and $\int_{\mathbb{R}} c^{0}(x) d x=0$. We now show that $c_{x}^{\text {int }}$ is bounded on $\mathbb{R} \times(0, T)$. Indeed, $c_{x}^{\text {int }}=\left(c^{0}\right)_{x} \star E(u)$. Similarly, we get

$$
\left|c_{x}^{\text {int }}\right|_{L^{\infty}(\mathbb{R})} \leq P\left|\left(c^{0}\right)_{x}\right|_{L^{1}(\mathbb{R})} .
$$

(3) We now prove the Lipschitz continuity in time of $c^{\text {int }}$. Let $x \in \mathbb{R}, 0<t, s<$ $T$. Then we have

$$
\begin{aligned}
\left|c^{\operatorname{int}}[u](x, t)-c^{\mathrm{int}}[u](x, s)\right| & =\left|c^{0} \star(E(u(\cdot, t))-E(u(\cdot, s)))(x)\right| \\
& \leq\left|c^{0}\right|_{L_{\mathrm{int}}^{\infty}(\mathbb{R})}|E(u(\cdot, t))-E(u(\cdot, s))|_{L_{\text {unif }}^{1}(\mathbb{R})} \\
& \leq \frac{4 P}{b}\left|c^{0}\right|_{L_{\mathrm{int}}^{\infty}(\mathbb{R})}|u(\cdot, t)-u(\cdot, s)|_{L^{\infty}(\mathbb{R})} \\
& \leq \frac{4 A P}{b}\left|c^{0}\right|_{L_{\mathrm{int}}^{\infty}(\mathbb{R})}|t-s|,
\end{aligned}
$$

where we have used successively Lemma 3.4, Proposition 3.1 (see Remark 3.2) and the Lipschitz continuity of $u$ we assumed to hold. 


\section{Proof of Theorem 1.3}

We prove Theorem 1.3 in two main steps. In a first step (see Subsection 4.1), we prove existence and uniqueness for short time, using a fixed point theorem. In a second step (see Subsection 4.2), we extend the result for all time by repeating the argument on successive time intervals. We need to recall Lemma 2.8 of Barles [7.

Lemma 4.1. Let $H$ be a continuous Hamiltonian. If $u \in C(\bar{\Omega} \times[0, T])$ is a subsolution (respectively, a supersolution) of the problem

$$
\frac{\partial u}{\partial t}+H(x, t, D u)=0 \quad \text { in } \quad \Omega \times(0, T)
$$

then $u$ is a subsolution (respectively, a supersolution) of the problem

$$
\frac{\partial u}{\partial t}+H\left(x, t, \frac{\partial u}{\partial x}\right)=0 \quad \text { in } \quad \Omega \times(0, T] .
$$

This lemma will be applied for $H\left(x, t, \frac{\partial u}{\partial x}\right)=c[u](x, t)\left|\frac{\partial u}{\partial x}\right|$, where $u$ is a solution on $(0, T), u \in W_{\text {loc }}^{1, \infty}(\mathbb{R} \times[0, T])$ and $c[u] \in C(\mathbb{R} \times[0, T])$.

4.1. Short time existence and uniqueness of the solution. For $c^{\text {ext }}$ satisfying (3) and $c^{0}$ satisfying (5), we denote

$$
K=\left|c^{\mathrm{ext}}\right|_{L^{\infty}(\mathbb{R})}+P\left|c^{0}\right|_{L^{1}(\mathbb{R})} .
$$

Consider four constants satisfying $0<b_{1}<b_{0}<B_{0}<B_{1}$, and for $T>0$, we set

$X_{T}=\left\{\begin{array}{l|l}u \in W_{\text {loc }}^{1, \infty}(\mathbb{R} \times[0, T)) & \begin{array}{l}u(x+1, t)=u(x, t)+P \quad \text { for }(x, t) \in \mathbb{R} \times[0, T), \\ 0<b_{1} \leq u_{x} \leq B_{1} \quad \text { a.e. on } \mathbb{R} \times[0, T), \\ \left|u_{t}\right| \leq K B_{1} \quad \text { a.e. on } \mathbb{R} \times[0, T)\end{array}\end{array}\right\}$.

Clearly, $X_{T}-P x$ is a closed set of the Banach space $W^{1, \infty}(\mathbb{R} \times[0, T))$. We want to establish that there exists a unique solution $u \in X_{T}$ of the problem

$$
\left\{\begin{array}{l}
\frac{\partial u}{\partial t}(x, t)=\left(c^{\mathrm{ext}}(x)+c^{0} \star(E(u(\cdot, t))-P \cdot)(x)\right) \frac{\partial u}{\partial x}(x, t) \quad \text { in } \mathbb{R} \times(0, T), \\
u(x, 0)=u^{0}(x) \text { on } \mathbb{R}
\end{array}\right.
$$

where $u^{0}$ satisfies assumptions (6). For any $u \in X_{T}$ such that $u(x, 0)=u^{0}(x)$, we consider the continuous viscosity solution $v$ of the problem (30)

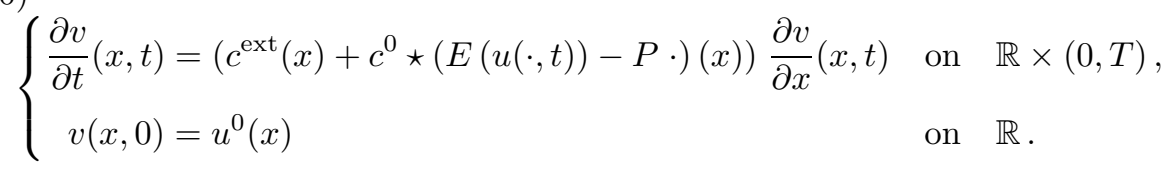

The main idea, in this section, is to show that the map

$$
\begin{aligned}
\varphi: & X_{T} \longrightarrow X_{T}, \\
& u \longmapsto \varphi(u)=v \quad \text { viscosity solution of (30) }
\end{aligned}
$$

is well defined and has a unique fixed point. 
We will first show that $\varphi$ is well defined for $T$ small enough, and then show that $\varphi$ is a contraction. Let us define

$$
L=\left|c_{x}^{\mathrm{ext}}\right|_{L^{\infty}(\mathbb{R})}+P\left|c_{x}^{0}\right|_{L^{1}(\mathbb{R})} \quad \text { and } \quad T^{*}=\frac{1}{L} \min \left(\ln \left(\frac{B_{1}}{B_{0}}\right), \ln \left(\frac{b_{0}}{b_{1}}\right)\right) .
$$

1) $\varphi\left(X_{T}\right) \subset X_{T}$ for $0<T \leq T^{*}$. We first remark that the solution $v$ of (30) is given by Proposition 2.2. Indeed this proposition applies because our initial condition satisfies its assumptions and the velocity $c(x, t)=$ $c^{\operatorname{ext}}(x)+c^{0} \star E(u(\cdot, t))(x)$ is in $W^{1, \infty}(\mathbb{R} \times[0, T])$ by Lemma 3.5 and the definition of $X_{T}$.

We will now check that $v \in X_{T}$ for $T$ small enough. By Lemma 3.5. assertion (2), we know that $\left|c^{\text {int }}\right|_{L^{\infty}(\mathbb{R})} \leq P\left|c^{0}\right|_{L^{1}(\mathbb{R})}$ and $\left|c_{x}^{\text {int }}\right|_{L^{\infty}(\mathbb{R})} \leq$ $P\left|\left(c^{0}\right)_{x}\right|_{L^{1}(\mathbb{R})}$. Therefore

$$
|c| \leq K=\left|c^{\mathrm{ext}}\right|_{L^{\infty}(\mathbb{R})}+P\left|c^{0}\right|_{L^{1}(\mathbb{R})}
$$

and

$$
\left|c_{x}\right| \leq L=\left|c_{x}^{\text {ext }}\right|_{L^{\infty}(\mathbb{R})}+P\left|c_{x}^{0}\right|_{L^{1}(\mathbb{R})} .
$$

By the a priori estimates for the eikonal equation (Proposition 2.2), we see that the function $v$ satisfies for a.e. $(x, t) \in \mathbb{R} \times[0, T)$

$$
\left\{\begin{array}{l}
\left|v_{x}(x, t)\right| \leq B_{0} e^{L t}=B(t), \\
v_{x}(x, t) \geq b_{0} e^{-L t}=b(t), \\
\left|v_{t}(x, t)\right| \leq|c|_{L^{\infty}(\mathbb{R} \times[0, T))} B(t)
\end{array}\right.
$$

and we have $B\left(T^{*}\right) \leq B_{1}$ and $b\left(T^{*}\right) \geq b_{1}$ with the definition of $T^{*}$ in (31).

By Lemma 3.5, assertion (2), we know that $c(x+1, t)=c(x, t)$. Let $w(x, t)=v(x+1, t)-P$. Then $w(x, 0)=u^{0}(x+1)-P=u^{0}(x)=v(x, 0)$. Then by the space periodicity of the velocity $c$ and the fact that the eikonal equation "does not see the constants", we deduce that $w$ is still a viscosity solution of (30). By the uniqueness of the solution we get that $w(x, t)=$ $v(x, t)$, and therefore $v(x+1, t)=v(x, t)+P$. We deduce that $v \in X_{T}$ if $T \leq T^{*}$.

2) $\varphi$ has a unique fixed point. Let us define $T_{0}$ by

$$
T_{0}=\min \left(\frac{1}{\left|c_{x}^{\text {ext }}\right|_{L^{\infty}(\mathbb{R})}+P\left|\left(c^{0}\right)_{x}\right|_{L^{1}(\mathbb{R})}}, \frac{1}{8 P\left|c^{0}\right|_{L_{\text {int }}^{\infty}(\mathbb{R})}}\right) \min \left(\ln \frac{b_{0}}{b_{1}}, \frac{b_{1}}{B_{1}}\right) .
$$

Indeed, the following proposition shows that $\varphi$ is a contraction.

Proposition 4.2 (Contraction). Let $v^{i}=\varphi\left(u^{i}\right)$ for $i=1,2$. If $u^{i} \in X_{T}$ for $i=1,2$, and if $\left|u^{2}-u^{1}\right|_{L^{\infty}(\mathbb{R} \times[0, T))} \leq P$, then

$$
\left|v^{2}-v^{1}\right|_{L^{\infty}(\mathbb{R} \times[0, T))} \leq \frac{1}{2}\left|u^{2}-u^{1}\right|_{L^{\infty}(\mathbb{R} \times[0, T))} \quad \text { for all } \quad T \in\left[0, T_{0}\right] .
$$

A corollary of this contraction property is

Proposition 4.3 (Short time existence and uniqueness of the solution). We assume that $c^{\text {ext }}$ and $c^{0}$ satisfy (3) and (5) and that $u^{0}$ satisfies (6). There then exists a unique continuous viscosity solution $u \in X_{T_{0}}$ of (29). 
To finish this subsection, we will first prove Proposition 4.3 and then Proposition 4.2

Proof of Proposition 4.3. Note that we can write

$$
\left[0, T_{0}\right]=\bigcup_{k=0, \ldots, N-1}\left[\frac{k T_{0}}{N}, \frac{(k+1) T_{0}}{N}\right],
$$

where $N$ will be large enough and fixed later. Let us denote $\tau_{k}=\left[\frac{k T_{0}}{N}, \frac{(k+1) T_{0}}{N}\right]$ for $k \in\{0, \ldots, N-1\}$.

Step 1. Let $u^{1}, u^{2} \in X_{T_{0}}$ such that $u^{1}(x, 0)=u^{2}(x, 0)=u^{0}(x)$. For all $t \in \tau_{0}$, and for all $x \in \mathbb{R}$, we compute

$$
\begin{aligned}
\left|u^{2}(x, t)-u^{1}(x, t)\right| & \leq\left|u^{2}(x, t)-u^{2}(x, 0)\right|+\left|u^{1}(x, 0)-u^{1}(x, t)\right| \\
& \leq 2 K B_{1}|t| \\
& \leq 2 K B_{1} \frac{T_{0}}{N} \\
& \leq 1 \leq P
\end{aligned}
$$

if we choose $N \geq 2 K B_{1} T_{0}$. Then Proposition 4.2 holds, i.e. $\varphi$ is a contraction on $X_{\frac{T_{0}}{N}}$. Since $X_{T_{0}}-P x$ is a closed subset of a Banach space then by the Banach-Picard fixed point theorem, there exists a unique solution $u \in X_{\frac{T_{0}}{N}}$ such that $u=\varphi(u)$, i.e. $u$ is a solution of (29) on $\tau_{0}=\left[0, \frac{T_{0}}{N}\right]$.

Step 2. First we remark that the solution $u \in X_{\frac{T_{0}}{N}}$ belongs to $W_{\text {loc }}^{1, \infty}\left(\mathbb{R} \times\left[0, \frac{T_{0}}{N}\right]\right)$ by the a priori bounds on $u_{x}$ and $u_{t}$ defining $X_{\frac{T_{0}}{N}}$. Second, we then apply Step 1 again with the new initial condition $u^{0}(\cdot):=u\left(\cdot, \frac{T_{0}}{N}\right)$ and get a solution $v \in X_{T_{0}}$. We then define

$$
u(\cdot, t)=v\left(\cdot, t-\frac{T_{0}}{N}\right) \quad \text { for } \quad t \in \tau_{1} .
$$

Third by construction, $u$ is a viscosity solution on $\left(0, \frac{T_{0}}{N}\right) \cup\left(\frac{T_{0}}{N}, \frac{2 T_{0}}{N}\right)$ and by Lemma 4.1 we see that it also satisfies the viscosity inequalities at time $\frac{T_{0}}{N}$, and therefore $u$ is a viscosity solution of (29) on $\left(0, \frac{2 T_{0}}{N}\right)$.

Step 3. We repeat the previous argument on the time intervals $\tau_{k}, k=$ $2, \ldots, N$, and get the existence of a viscosity solution $u$ of (29) on the time interval $\left(0, T_{0}\right)$.

Step 4. Uniqueness. Let us assume that we have two solutions $u^{1}$ and $u^{2}$ of (29) on $\left(0, T_{0}\right)$, with $u^{1} \neq u^{2}$ and let us define $T_{0}^{*}<T_{0}$ such that $u^{1}=u^{2}$ on $\left[0, T_{0}^{*}\right]$ and

$\forall \delta>0, \exists t_{\delta} \in\left[T_{0}^{*}, T_{0}^{*}+\delta\right] \cap\left[T_{0}^{*}, T_{0}\right]$ such that $u^{2}\left(\cdot, t_{\delta}\right) \neq u^{1}\left(\cdot, t_{\delta}\right)$.

Again applying Step 1 with initial condition $u^{0}(\cdot):=u^{1}\left(\cdot, T_{0}^{*}\right)=u^{2}\left(\cdot, T_{0}^{*}\right)$, 
we get by the contraction property that $\left(u^{i}=\varphi\left(u^{i}\right), i=1,2\right)$

$$
\left|u^{2}-u^{1}\right|_{L^{\infty}\left(\mathbb{R} \times\left[0, T_{0}^{*}+\delta\right)\right)} \leq \frac{1}{2}\left|u^{2}-u^{1}\right|_{L^{\infty}\left(\mathbb{R} \times\left[0, T_{0}^{*}+\delta\right)\right)}
$$

for $\delta \leq \frac{T_{0}}{N}$ and $T_{0}^{*}+\delta \leq T_{0}$ (using (33)). Contradiction.

Proof of Proposition 4.2, Let $v^{i}=\varphi\left(u^{i}\right)$ for $i=1,2$. By the stability result (Proposition 2.3), we have

$$
\begin{aligned}
& \left|v^{1}-v^{2}\right|_{L^{\infty}(\mathbb{R} \times[0, T])} \\
& \leq \int_{0}^{T}\left|c^{2}(\cdot, s)-c^{1}(\cdot, s)\right|_{L^{\infty}(\mathbb{R})} \max \left(\left|v_{x}^{1}(\cdot, s)\right|_{L^{\infty}(\mathbb{R})},\left|v_{x}^{2}(\cdot, s)\right|_{L^{\infty}(\mathbb{R})}\right) d s,
\end{aligned}
$$

where $c^{i}(x, t)=c^{\text {ext }}(x)+\left(c^{0} \star E\left(u^{i}(\cdot, t)\right)\right)(x)$. By Lemma 3.4, we have

$$
\left|c^{2}-c^{1}\right|_{L^{\infty}(\mathbb{R} \times[0, T))} \leq\left|c^{0}\right|_{L_{\text {int }}^{\infty}(\mathbb{R})}\left|E\left(u^{2}\right)-E\left(u^{1}\right)\right|_{L^{\infty}\left([0, T), L_{\text {unif }}^{1}(\mathbb{R})\right)} .
$$

By Proposition 3.1 and Remark 3.2, we know that

$$
\left|E\left(u^{2}\right)-E\left(u^{1}\right)\right|_{L^{\infty}\left([0, T), L_{\text {unif }}^{1}(\mathbb{R})\right)} \leq \frac{4 P}{b}\left|u^{2}-u^{1}\right|_{L^{\infty}(\mathbb{R} \times[0, T))},
$$

then combining Proposition 4.2 and Lemma 3.4, we obtain

$$
\left|v^{1}-v^{2}\right|_{L^{\infty}(\mathbb{R} \times[0, T])} \leq 4 P T \frac{B}{b}\left|c^{0}\right|_{L_{\text {int }}^{\infty}(\mathbb{R})}\left|u^{2}-u^{1}\right|_{L^{\infty}(\mathbb{R} \times[0, T))} .
$$

We set $T^{* *}=\frac{1}{8 P\left|c^{0}\right|_{L_{\text {int }}^{\infty}(\mathbb{R})}} \frac{b}{B}$. For $T_{0}=\inf \left(T^{*}, T^{* *}\right)$, the following holds: for $T \leq T_{0}$ :

$$
\left|v^{2}-v^{1}\right|_{L^{\infty}(\mathbb{R} \times[0, T))} \leq \frac{1}{2}\left|u^{2}-u^{1}\right|_{L^{\infty}(\mathbb{R} \times[0, T))} .
$$

\subsection{Long time existence of the viscosity solution: Proof of Theorem $\mathbf{1 . 3}$.}

Proof of Theorem 1.3. We will repeat this short time result on a sequence of time intervals of lengths $T_{n}$ decreasing to zero, such that $\sum_{n \in \mathbb{N}} T_{n}=+\infty$. We will do the proof in three steps.

Step 1. We rephrase the result of Proposition 4.3. We proved in the previous subsection that given an initial data $u^{0}$ such that

$$
0<b_{0} \leq u_{x}^{0} \leq B_{0}
$$

and $b_{1}, B_{1}$ (which will be specified later) such that

$$
0<b_{1}<b_{0}<B_{0}<B_{1} \quad \text { and } \quad \frac{b_{0}}{b_{1}}=\frac{B_{1}}{B_{0}}
$$

there exists a unique viscosity solution $u$ of problem (29) up to time $T_{0}$ satisfying

$$
0<b_{1} \leq u_{x} \leq B_{1} \quad \text { on } \quad \mathbb{R} \times\left[0, T_{0}\right),
$$

where $T_{0}$ is defined by

$$
T_{0}=A \min \left\{\ln \mu_{0}, \frac{1}{\mu_{0}^{2}} \bar{b}_{0}\right\},
$$


where

$$
\left\{\begin{aligned}
A & =\min \left\{\frac{1}{\left|c_{x}^{\text {ext }}\right|_{L^{\infty}(\mathbb{R})}+P\left|c_{x}^{0}\right|_{L^{1}(\mathbb{R})}}, \frac{1}{8 P\left|c^{0}\right|_{L_{\text {int }}^{\infty}(\mathbb{R})}}\right\}, \\
\mu_{0} & =\frac{b_{0}}{b_{1}}=\frac{B_{1}}{B_{0}}>1, \text { and } \\
\bar{b}_{0} & =\frac{b_{0}}{B_{0}}<1 .
\end{aligned}\right.
$$

For given $b_{0}$ and $B_{0}$, in order to equalize the two terms in the infimum of (34), we choose $\mu_{0}$ such that $\ln \mu_{0}=\frac{1}{\mu_{0}^{2}} \bar{b}_{0}$, in other words, $\mu_{0}$ is fixed by the relation

$$
\bar{b}_{0}=\mu_{0}^{2} \ln \mu_{0}
$$

and it determinates $b_{1}$ and $B_{1}$ as a function of $b_{0}, B_{0}$. Therefore, we have

$$
T_{0}=A \ln \mu_{0} .
$$

Step 2. Definition of the recurrence. We apply successively this reasoning on time intervals of length $T_{n}$ which will be specified below. So, for $n \geq 1$, for $b_{n+1}, B_{n+1}$ (which will be specified later) there exists a unique solution of the problem (29) up to time $T_{0}+T_{1}+\cdots+T_{n}$, where

$$
\begin{gathered}
\mu_{n}=\frac{b_{n}}{b_{n+1}}=\frac{B_{n+1}}{B_{n}}>1 \text { for } 0<b_{n+1}<b_{n}<B_{n}<B_{n+1}, \\
\bar{b}_{n}=\frac{b_{n}}{B_{n}}<1 \text { and } \mu_{n} \quad \text { is fixed by } \quad \bar{b}_{n}=\mu_{n}^{2} \ln \mu_{n}, \\
T_{n}=A \ln \mu_{n} .
\end{gathered}
$$

\section{Step 3. Divergence of the series $\sum_{n \in \mathbb{N}} T_{n}$.}

Proposition 4.4. With previous notation and the choice of the sequence $\left(\mu_{n}\right)_{n}$, the series $\sum_{n \in \mathbb{N}} T_{n}$ diverges.

This ends the proof of Theorem 1.3 .

In the rest of this subsection, we will prove Proposition 4.4. Before proving this proposition we need preliminary results. First, we remark by (36) that

$$
\bar{b}_{n+1}=\frac{b_{n+1}}{B_{n+1}}=\frac{1}{\mu_{n}^{2}} \bar{b}_{n},
$$

and then by (37) and (38) we get

$$
\ln \mu_{n}=\mu_{n+1}^{2} \ln \mu_{n+1} \quad \text { and } \quad T_{n}=A \ln \mu_{n} .
$$

The recurrence relation defining the sequence $\left(\mu_{n}\right)_{n}$ can be inverted as $\mu_{n+1}=$ $G\left(\mu_{n}\right)$ with $\mu_{n}>1$. Introducing

$$
\varepsilon_{n}=\mu_{n}-1>0,
$$

we can rewrite $\mu_{n+1}=G\left(\mu_{n}\right)$ as

$$
\varepsilon_{n+1}=F\left(\varepsilon_{n}\right),
$$

where by the implicit function theorem $F \in C^{2}\left(\left(-\frac{1}{2 e},+\infty\right) ; \mathbb{R}\right)$ and satisfies

$$
F(0)=0, F^{\prime}(0)=1, F^{\prime \prime}(0)=-4 \text {, and } F^{\prime}>0 .
$$

We have the following lemma. 
Lemma 4.5 (Subsolution for the sequence). Let $F \in C^{2}([0,+\infty) ; \mathbb{R})$ satisfying (41). For $a \geq 0$, let

$$
\sigma(a)=\int_{0}^{1} d t\left(F^{\prime \prime}(t a)-F^{\prime \prime}(0)\right)(1-t)
$$

and

$$
a_{0}=\sup \left\{a \geq 0: \inf _{[0, a]} \sigma \geq-2\right\} .
$$

Then $a_{0}>0$. Let $\rho_{a}(t)$ be the solution of

$$
\left\{\begin{array}{l}
\rho_{a}^{\prime}(t)=-4 \rho_{a}^{2}(t) \\
\rho_{a}(0)=a
\end{array}\right.
$$

If $a \in\left[0, a_{0}\right]$, then for all $t \geq 0$, we have

$$
\rho_{a}(t+1) \leq F\left(\rho_{a}(t)\right) .
$$

Corollary 4.6 (A lower bound on the sequence $\left.\left(\varepsilon_{n}\right)_{n}\right)$. Under the assumptions of Lemma 4.5, we consider a sequence $\left(\varepsilon_{n}\right)_{n}>0$ satisfying $\varepsilon_{n+1}=F\left(\varepsilon_{n}\right)$. If for an integer $k$, we have $0<\varepsilon_{k} \leq a_{0}$, then for all $n \geq 0$ we have

$$
\varepsilon_{k+n} \geq \rho(n),
$$

where $\rho=\rho_{\varepsilon_{k}}$.

Proof of Corollary 4.6. Since $\rho$ is decreasing in $t\left(\rho^{\prime}(t)<0\right), \rho(n) \leq \rho(0)=\varepsilon_{k}$. Applying (43) and using the fact that $F$ is increasing, we get

$$
\rho(n) \leq F(\rho(n-1)) \leq \cdots \leq F^{n}(\rho(0))=F^{n}\left(\varepsilon_{k}\right)=\varepsilon_{k+n} .
$$

Proof of Lemma 4.5. We set $\phi(t)=F(t a)$. Using the Taylor formula with integral remainder, we have

$$
\begin{aligned}
\phi(1) & =\phi(0)+\phi^{\prime}(0)+\int_{0}^{1} d t \phi^{\prime \prime}(t)(1-t) \\
& =\phi(0)+\phi^{\prime}(0)+\frac{\phi^{\prime \prime}(0)}{2}+\int_{0}^{1} d t\left(\phi^{\prime \prime}(t)-\phi^{\prime \prime}(0)\right)(1-t)
\end{aligned}
$$

with $\phi^{\prime \prime}(t)=F^{\prime \prime}(t a) a^{2}$. Then setting $\sigma(a)=\int_{0}^{1}\left(F^{\prime \prime}(t a)-F^{\prime \prime}(0)\right)(1-t) d t$, we get $\sigma \in C^{0}([0,+\infty), \mathbb{R})$ and $F(a)=a-2 a^{2}+a^{2} \sigma(a)$. Thus,

$$
\begin{aligned}
F(\rho(t))-\rho(t+1) & =\rho(t)-2 \rho^{2}(t)+\rho^{2}(t) \sigma(\rho(t))-\rho(t+1) \\
& \geq \rho(t)-\rho(t+1)-4 \rho^{2}(t)
\end{aligned}
$$

because $\rho(t) \in\left[0, a_{0}\right]$ from the assumption of the lemma (and the fact that $\rho$ is decreasing in $t$ ), which guarantees $\sigma(\rho(t)) \geq-2$. We now estimate

$$
\begin{aligned}
\rho(t)-\rho(t+1) & =\int_{t}^{t+1}-\rho^{\prime}(s) d s \\
& =\int_{t}^{t+1} 4 \rho^{2}(s) d s \quad \geq 4 \rho^{2}(t) .
\end{aligned}
$$

We deduce that $F(\rho(t))-\rho(t+1) \geq 0$. 
Proof of Proposition 4.4. Let us first remark that $\sum_{n \geq 0} T_{n}=\sum_{n \geq 0} \ln \mu_{n}$ $=\ln \prod_{n \geq 0}\left(1+\varepsilon_{n}\right) \geq \ln \left(1+\sum_{n \geq 0} \varepsilon_{n}\right)$. We will now show that $\sum_{n \geq 0} \varepsilon_{n}$ diverges. If it is not the case, then $\varepsilon_{k} \longrightarrow 0$ when $k \rightarrow \infty$, and so for $k$ large enough we have $\varepsilon_{k} \leq a_{0}$. Therefore, by Corollary 4.6. we know that $\varepsilon_{k+n} \geq \rho(n-1)$ for all $n \in \mathbb{N}$ and $\rho(t)=\frac{1}{\frac{1}{a}+4 t}$. We deduce that

$$
\sum_{n \geq 0} \varepsilon_{n} \geq \sum_{n \geq 1} \rho(n) \geq \int_{1}^{+\infty} \rho(t) d t=\int_{1}^{+\infty} \frac{1}{\frac{1}{a}+4 t} d t=+\infty .
$$

Then the series $\sum_{n \geq 0} \varepsilon_{n}$ diverges and $\sum_{n \geq 0} T_{n}$ also diverges.

\section{Preliminary Results for the discrete local Problem}

As explained in Subsection 1.2. we construct a numerical scheme for the nonlocal equation by explicitly discretising the time variable by an Euler scheme and the space variable by an upwind scheme. We first study the case of a local equation whose gradient satisfies $\frac{\partial u}{\partial x} \geq 0$. This leads us to study the following local transport equation:

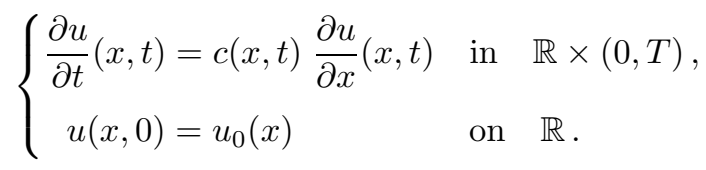

Given a mesh size $\Delta x, \Delta t$ and a lattice $I_{d}=\left\{(i \Delta x, n \Delta t) ; i \in \mathbb{Z}, n \leq \frac{T}{\Delta t}\right\},\left(x_{i}, t_{n}\right)$ denotes the node $(i \Delta x, n \Delta t)$ and $v^{n}=\left(v_{i}^{n}\right)_{i}$ the values of the numerical approximation of the continuous solution $u\left(x_{i}, t_{n}\right)$. We consider an explicit Euler scheme in time, i.e.

$$
v_{i}^{n+1}=v_{i}^{n}+\Delta t H_{d}\left(v^{n}, i\right),
$$

where the discrete Hamiltonian is chosen so that the scheme is upwind; precisely we choose

$$
H_{d}\left(v^{n}, i\right)=\left\{\begin{array}{cl}
c_{i}^{n} D_{x}^{+} v_{i}^{n} & \text { if } c_{i}^{n} \geq 0 \\
c_{i}^{n} D_{x}^{-} v_{i}^{n} & \text { if } c_{i}^{n}<0
\end{array}\right.
$$

with

$$
D_{x}^{+} v_{i}^{n}=\frac{v_{i+1}^{n}-v_{i}^{n}}{\Delta x}, \quad D_{x}^{-} v_{i}^{n}=\frac{v_{i}^{n}-v_{i-1}^{n}}{\Delta x},
$$

and $c_{i}^{n}$ is the discrete velocity.

We assume the following CFL condition for the local problem

$$
\Delta t \leq \frac{\Delta x}{\sup _{i, n}\left|c_{i}^{n}\right|} .
$$

For the reader's convenience, we recall some useful results proved in 2, 3. We first recall a discrete gradient estimate from above whose proof is given in [2].

Lemma 5.1 (Discrete gradient estimate from above). If for some $B^{0}>0$ we have $\left|\frac{v_{i+1}^{0}-v_{i}^{0}}{\Delta x}\right| \leq B^{0}, \forall i \in \mathbb{Z}$, and $B^{n+1}=B^{n}\left(1+2 \Delta t \sup _{j \in \mathbb{Z}}\left|\frac{c_{j+1}^{n}-c_{j}^{n}}{\Delta x}\right|\right)$, then

$$
\left|\frac{v_{i+1}^{n}-v_{i}^{n}}{\Delta x}\right| \leq B^{n} \forall i \in \mathbb{Z}, \forall n \in \mathbb{N} .
$$

In the following, we also need a discrete gradient estimate from below. 
Lemma 5.2 (Discrete gradient estimate from below). If for some $b^{0}>0$ we have $\frac{v_{i+1}^{0}-v_{i}^{0}}{\Delta x} \geq b^{0}, \forall i \in \mathbb{Z}$, and $b^{n+1}=b^{n}\left(1-2 \Delta t \sup _{j \in \mathbb{Z}}\left|\frac{c_{j+1}^{n}-c_{j}^{n}}{\Delta x}\right|\right)$ with

$$
\Delta t<\frac{1}{2 \sup _{j \in \mathbb{Z}}\left|\frac{c_{j+1}^{n}-c_{j}^{n}}{\Delta x}\right|},
$$

then

$$
\frac{v_{i+1}^{n}-v_{i}^{n}}{\Delta x} \geq b^{n}, \forall i \in \mathbb{Z}, \forall n \in \mathbb{N} .
$$

Proof of Lemma 5.2. First, let us remark that $b^{n} \geq 0$ because of the condition $\Delta t<\frac{1}{2 \sup _{j \in \mathbb{Z}}\left|\frac{c_{j+1}^{n}-c_{j}^{n}}{\Delta x}\right|}$. Let $w_{i}^{n}=v_{i-1}^{n}+b^{n} \Delta x$. By assumption, we have $w_{i}^{n} \leq$ $v_{i}^{n}, \forall i \in \mathbb{Z}$. In order to show that $w_{i}^{n+1} \leq v_{i}^{n+1}$ for all $i \in \mathbb{Z}$, we check that $w^{n}$ is a discrete subsolution i.e. $w_{i}^{n+1}-\left(w_{i}^{n}+\Delta t H_{d}\left(w^{n}, i\right)\right) \leq 0$. Indeed,

$$
\begin{aligned}
& w_{i}^{n+1}-\left(w_{i}^{n}+\Delta t H_{d}\left(w^{n}, i\right)\right) \\
& =v_{i-1}^{n+1}+b^{n+1} \Delta x-\left(v_{i-1}^{n}+b^{n} \Delta x+\Delta t H_{d}\left(w^{n}, i\right)\right) \\
& =\left(b^{n+1}-b^{n}\right) \Delta x+\Delta t\left(H_{d}\left(v^{n}, i-1\right)-H_{d}\left(w^{n}, i\right)\right) \\
& =\left(b^{n+1}-b^{n}\right) \Delta x+\Delta t\left(H_{d}\left(v^{n}, i-1\right)-H_{d}\left(v_{\cdot-1}^{n}, i\right)\right) .
\end{aligned}
$$

If $c_{i}^{n}$ and $c_{i-1}^{n}$ have the same sign, we assume that they are non-negative (the proof is similar when they are non-positive), then

$$
\begin{aligned}
& w_{i}^{n+1}-\left(w_{i}^{n}+\Delta t H_{d}\left(v^{n}, i\right)\right) \\
& =-2 b^{n} \Delta t \Delta x \sup _{j \in \mathbb{Z}}\left|\frac{c_{j}^{n}-c_{j-1}^{n}}{\Delta x}\right|+\Delta t\left(c_{i-1}^{n}-c_{i}^{n}\right) D_{x}^{+} v_{i}^{n} \\
& =-2 b^{n} \Delta t \sup _{j \in \mathbb{Z}}\left|c_{j}^{n}-c_{j-1}^{n}\right|-\left(c_{i}^{n}-c_{i-1}^{n}\right) \Delta t D_{x}^{+} v_{i-1}^{n} \\
& \leq-b^{n} \Delta t\left(2 \sup _{j \in \mathbb{Z}}\left|c_{j}^{n}-c_{j-1}^{n}\right|+c_{i}^{n}-c_{i-1}^{n}\right) \\
& \leq 0 .
\end{aligned}
$$

Therefore, $w^{n}$ is a discrete subsolution and then $w_{i}^{n+1} \leq v_{i}^{n+1}$ for all $i \in \mathbb{Z}$. If $c_{i}^{n}$ and $c_{i-1}^{n}$ do not have the same sign (we refer the reader to the end of the proof of Lemma 5.1 in [3]), the conclusion prevails because of the following estimate for $a, b \geq 0$ :

$$
\begin{aligned}
\left|c_{i}^{n} a-c_{i-1}^{n} b\right| & \leq \max (a, b) \max \left(\left|c_{i}^{n}\right|,\left|c_{i-1}^{n}\right|\right) \\
& \leq \max (a, b)\left|c_{i}^{n}-c_{i-1}^{n}\right| \\
& \leq \max (a, b)\left|\frac{c_{i}^{n}-c_{i-1}^{n}}{\Delta x}\right| \Delta x
\end{aligned}
$$

This achieves the proof of Lemma 5.2

We introduce the grid $I_{d}^{T}=\left\{(i \Delta x, n \Delta t) ; i \in \mathbb{Z}, n \leq N_{T}=\frac{T}{\Delta t}\right\}$. We recall the following numerical stability result, whose proof is given in $[3,2]$.

Proposition 5.3 (Numerical stability). We consider $v^{1, n}$ and $v^{2, n}$ two numerical solutions of the following monotone scheme (with the same initial condition)

$$
v_{i}^{l, n+1}=v_{i}^{l, n}+\Delta t H_{d}\left(v^{l, n}, i\right),
$$


where

$$
H_{d}\left(v^{l, n}, i\right)=\left\{\begin{array}{cc}
c_{i}^{l, n} D_{x}^{+} v_{i}^{l, n} & \text { if } c_{i}^{l, n} \geq 0, \\
c_{i}^{l, n} D_{x}^{-} v_{i}^{l, n} & \text { if } c_{i}^{l, n}<0,
\end{array} \quad \text { for } l=1,2, \forall i \in \mathbb{Z}, \forall n \in \mathbb{N} .\right.
$$

Then there exists a constant $C>0$, depending on the discrete gradient estimates on $v^{1}$ and $v^{2}$, such that

$$
\sup _{I_{d}^{T}}\left|v_{i}^{1, n+1}-v_{i}^{2, n+1}\right| \leq C T \sup _{I_{d}^{T}}\left|c_{i}^{1, n}-c^{2, n}\right| .
$$

\section{Preliminary Result For the Discrete nON-LOCAl PROBlem}

We will prove the analogue of Proposition 3.1 in the framework of discrete solutions. We will use this result in Section 7.

Proposition 6.1 (Estimate of the difference of integer parts in the discrete case). Consider a discrete function $v^{1}$ such that

$$
v_{i+K}^{1}=v_{i}^{1}+P \text { where } P \in \mathbb{N} \backslash\{0\} \quad \text { and } \quad K=\frac{1}{\Delta x} \in \mathbb{N} \backslash\{0\} .
$$

Assume that there exist two constants $B \geq b>0$ such that for every $i \in \mathbb{Z}$ we have

$$
b \leq \frac{v_{i+1}^{1}-v_{i}^{1}}{\Delta x} \leq B .
$$

Then for all discrete functions $v^{2}$ satisfying (150), we get

$$
\begin{aligned}
\sup _{i \in \mathbb{Z}} \sum_{j \in J_{i}}=[i, i+K[ & \left|E\left(v_{j}^{2}\right)-E\left(v_{j}^{1}\right)\right| \Delta x \\
& \leq 2\left(P+\sup _{i \in \mathbb{Z}}\left|v_{i}^{2}-v_{i}^{1}\right|\right)\left(\frac{1}{b} \sup _{i \in \mathbb{Z}}\left|v_{i}^{2}-v_{i}^{1}\right|+\Delta x\right) .
\end{aligned}
$$

Remark 6.2. Note that if $\sup _{i \in \mathbb{Z}}\left|v_{i}^{2}-v_{i}^{1}\right| \leq 1$, then

$$
\sup _{i \in \mathbb{Z}} \sum_{j \in J_{i}=[i, i+K[}\left|E\left(v_{j}^{2}\right)-E\left(v_{j}^{1}\right)\right| \Delta x \leq 4 P\left(\frac{1}{b} \sup _{i \in \mathbb{Z}}\left|v_{i}^{2}-v_{i}^{1}\right|+\Delta x\right) .
$$

This result is the discrete analogue of Proposition 3.1. This is also the generalization of Lemma 5.5 in 3 to the case of several dislocations where the characteristic function $v^{l}>0$ is replaced with the floor part $E\left(v^{l}\right)$.

For the proof of Proposition 6.1 we need to introduce the following notation.

We denote $\Lambda^{\prime}=\sup _{j \in \mathbb{Z}}\left|v_{j}^{2}-v_{j}^{1}\right|$, and we assume that $\Lambda^{\prime} \in(0,+\infty)$ (other cases are trivial). For $m \in \mathbb{Z}$ and for $l=1,2$, we denote $E_{m}^{l}=\left\{j \in \mathbb{Z}: v_{j}^{l}<m+1\right\}$. First, we remark that since $\frac{v_{i+1}^{1}-v_{i}^{1}}{\Delta x} \geq b>0$, there exists the greatest integer $j_{0} \in \mathbb{Z}$ such that $v_{j_{0}}^{1}<m+1$ and we have $E_{m}^{1}=\left\{j \in \mathbb{Z}: j \leq j_{0}\right\}$. We will use the following lemma for the proof of Proposition 6.1.

Lemma 6.3 (Estimate for the distance between the sets $E_{m}^{1}$ and $E_{m}^{2}$ ). Under the notation above and the assumptions of Proposition 6.1, we have

$$
E_{m}^{1}-E\left(\frac{\Lambda^{\prime}}{b \Delta x}\right)-1 \subset E_{m}^{2} \subset E_{m}^{1}+E\left(\frac{\Lambda^{\prime}}{b \Delta x}\right)+1 .
$$


Proof of Lemma 6.3. The main idea in this proof is to use the discrete gradient estimate from below. We will estimate in two steps the distance between $E_{m}^{1}$ and $E_{m}^{2}$.

Step 1. We have $\mathbf{E}_{\mathbf{m}}^{1}-\mathbf{E}\left(\frac{\Lambda^{\prime}}{\mathbf{b} \mathbf{\Delta x}}\right)-\mathbf{1} \subset \mathbf{E}_{\mathbf{m}}^{2}$. Indeed, let $j \in E_{m}^{1}-E\left(\frac{\Lambda^{\prime}}{b \Delta x}\right)-1$.

Then, $j \leq j_{0}-E\left(\frac{\Lambda^{\prime}}{b \Delta x}\right)-1$ i.e. $j_{0}-j \geq E\left(\frac{\Lambda^{\prime}}{b \Delta x}\right)+1>\frac{\Lambda^{\prime}}{b \Delta x}$ i.e. $\left(j_{0}-j\right) b \Delta x \geq \Lambda^{\prime}$. Since $\frac{v_{j_{0}}^{1}-v_{j}^{1}}{\left(j_{0}-j\right) \Delta x} \geq b$ and $j_{0}-j>0$, we have

$$
v_{j_{0}}^{1}-v_{j}^{1} \geq\left(j_{0}-j\right) b \Delta x>\Lambda^{\prime},
$$

which implies (by definition of $\Lambda^{\prime}$ )

$$
m+1>v_{j_{0}}^{1}>v_{j}^{1}+\Lambda^{\prime} \geq v_{j}^{2}
$$

and therefore $v_{j}^{2}<m+1$. Thus, $j \in E_{m}^{2}$.

Step 2. We have $\mathbf{E}_{\mathbf{m}}^{2} \subset \mathbf{E}_{\mathbf{m}}^{1}+\mathbf{E}\left(\frac{\boldsymbol{\Lambda}^{\prime}}{\mathbf{b} \mathbf{\Delta x}}\right)+1$. Similarly, considering $j \in$ $\left(E_{m}^{1}+E\left(\frac{\Lambda^{\prime}}{b \Delta x}\right)+1\right)^{c}$ we prove that $j \in\left(E_{m}^{2}\right)^{c}$. Indeed, $j>j_{0}+E\left(\frac{\Lambda^{\prime}}{b \Delta x}\right)+1$, then $j \geq j_{0}+1+E\left(\frac{\Lambda^{\prime}}{b \Delta x}\right)+1$ implies $j-\left(j_{0}+1\right) \geq E\left(\frac{\Lambda^{\prime}}{b \Delta x}\right)+1>\frac{\Lambda^{\prime}}{b \Delta x}$, i.e. $\left(j-\left(j_{0}+1\right)\right) b \Delta x>\Lambda^{\prime}$. Since $\frac{v_{j}^{1}-v_{j_{0}+1}^{1}}{\left(j-\left(j_{0}+1\right)\right) \Delta x} \geq b$ and $j-\left(j_{0}+1\right)>0$, we have

$$
v_{j}^{1}-v_{j_{0}+1}^{1} \geq\left(j-\left(j_{0}+1\right)\right) b \Delta x>\Lambda^{\prime},
$$

which implies (by definition of $\Lambda^{\prime}$ )

$$
v_{j}^{2} \geq v_{j}^{1}-\Lambda^{\prime}>v_{j_{0}+1}^{1} \geq m+1 .
$$

Then we have $j \in\left(E_{m}^{2}\right)^{c}$ and therefore $\left(E_{m}^{1}+E\left(\frac{\Lambda^{\prime}}{b \Delta x}\right)+1\right)^{c} \subset\left(E_{m}^{2}\right)^{c}$.

Proof of Proposition 6.1. The main idea in this proof is to bound the quantity $\left|E\left(v_{j}^{2}\right)-E\left(v_{j}^{1}\right)\right|$ by the characteristic functions the sets $E_{m}^{2} \triangle E_{m}^{1}$. We then bound the discrete analogue of its $L_{\text {unif }}^{1}$-norm.

From the definition of $E_{m}^{l}$, for $l=1,2$, we remark that $E_{m-1}^{l} \subset E_{m}^{l}$. Then $E\left(v_{j}^{l}\right)=m$ for any $j \in E_{m}^{l} \backslash E_{m-1}^{l}$, for $l=1,2$. We define

$$
1_{A}(j)= \begin{cases}1 & \text { if } j \in A \subset \mathbb{Z} \\ 0 & \text { if not. }\end{cases}
$$

Then we can write

$$
E\left(v_{j}^{l}\right)=\sum_{m \in \mathbb{N}} 1_{\left(E_{m}^{l}\right)^{c}}(j)-\sum_{m \in \mathbb{Z} \backslash \mathbb{N}} 1_{E_{m}^{l}}(j) .
$$

Similarly to (22) in the proof of Proposition 3.1 we get

$$
\left|E\left(v_{j}^{2}\right)-E\left(v_{j}^{1}\right)\right| \leq \sum_{m \in \mathbb{Z}} 1_{E_{m}^{2} \Delta E_{m}^{1}}(j) .
$$


Let us fix $i \in \mathbb{Z}$ and define $J_{i}=\left[i, i+K\left[\right.\right.$. Then the discrete analogue of $L_{\text {unif }}^{1}$-norm of $E\left(v^{2}\right)-E\left(v^{1}\right)$ satisfies

$$
\begin{aligned}
\sum_{j \in J_{i}}\left|E\left(v_{j}^{2}\right)-E\left(v_{j}^{1}\right)\right| & \leq \sum_{j \in \mathbb{Z}} \sum_{m \in \mathbb{Z}} 1_{J_{i}}(j) 1_{\left(E_{m}^{2} \Delta E_{m}^{1}\right)}(j) \\
& \leq \sum_{m \in \mathbb{Z}}\left|J_{i} \cap\left(E_{m}^{2} \Delta E_{m}^{1}\right)\right| \\
& \leq 2 N^{\prime}\left(\frac{\Lambda^{\prime}}{b \Delta x}+1\right),
\end{aligned}
$$

where $N^{\prime}=\operatorname{Card}\left\{m \in \mathbb{Z},\left|J_{i} \cap\left(E_{m}^{2} \Delta E_{m}^{1}\right)\right| \neq 0\right\}$ and where we have used the fact that the measure $\left|E_{m}^{2} \Delta E_{m}^{1}\right| \leq 2\left(E\left(\frac{\Lambda^{\prime}}{b \Delta x}\right)+1\right) \leq 2\left(\frac{\Lambda^{\prime}}{b \Delta x}+1\right)$. Similarly to (24) in the proof of Proposition 3.1, we get

$$
N^{\prime} \leq P+\sup _{i \in \mathbb{Z}}\left|v_{i}^{2}-v_{i}^{1}\right|
$$

We then conclude that

$$
\sup _{i \in \mathbb{Z}} \sum_{j \in J_{i}}\left|E\left(v_{j}^{2}\right)-E\left(v_{j}^{1}\right)\right| \Delta x \leq 2\left(P+\sup _{i \in \mathbb{Z}}\left|v_{i}^{2}-v_{i}^{1}\right|\right)\left(\frac{1}{b} \sup _{i \in \mathbb{Z}}\left|v_{i}^{2}-v_{i}^{1}\right|+\Delta x\right) .
$$

\section{Proof of Theorem 1.5}

In this section, we first recall how to get an error estimate between the continuous solution and the discrete solution for a general non-local transport equation for some $\bar{T}>0$. We are inspired by the work of $[2$.

7.1. An abstract error estimate. We consider the continuous viscosity solution $u$ of a general non-local transport equation

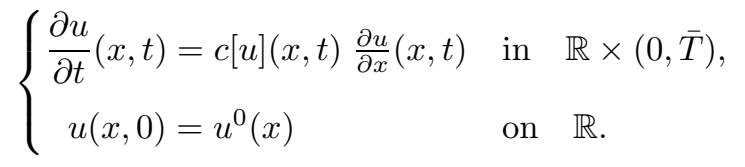

We recall that the non-local velocity $c[u]$ belongs to $L^{\infty}\left((0, \bar{T}), W^{1, \infty}(\mathbb{R})\right)$ and that the solution $u$ is Lipschitz continuous. We will consider a discrete solution $v$ satisfying

$$
v=G^{\Delta} \circ c^{\Delta}(v)
$$

where this abstract scheme will be made precise below.

For $0<T \leq \bar{T}$ and given a mesh $\Delta=(\Delta x, \Delta t)$, we denote $E_{T}^{\Delta}=\mathbb{R}^{\mathbb{Z} \times\left\{0, \ldots, N_{T}\right\}}$, $N_{T}$ is the floor part of $\frac{T}{\Delta t}$, the space of discrete functions defined on

$$
I_{d}^{T}=\left\{(i \Delta x, n \Delta t), i \in \mathbb{Z}, n \leq N_{T}\right\} .
$$

We consider two subsets of $E_{T}^{\Delta}$ :

$$
U_{T}^{\Delta}=\left\{w \in E_{T}^{\Delta} \text { such that }\left|\frac{w_{i+1}^{n}-w_{i}^{n}}{\Delta x}\right| \leq B^{0} e^{L T} \quad \text { and } \quad \frac{w_{i+1}^{n}-w_{i}^{n}}{\Delta x} \geq b^{0} e^{-L T}\right\}
$$

and

$V_{T}^{\Delta}=\left\{c \in E_{T}^{\Delta}\right.$ such that $\left|c_{i}^{n}\right| \leq K \quad$ and $\left.\quad\left|\frac{c_{i+1}^{n}-c_{i}^{n}}{\Delta x}\right| \leq L \forall i \in \mathbb{Z}, \forall n \leq N_{T}\right\}$. 
We also consider two operators:

$$
\begin{aligned}
& G^{\Delta}: V_{T}^{\Delta} \longrightarrow U_{T}^{\Delta} \quad \text { and } \quad c^{\Delta}: U_{T}^{\Delta} \longrightarrow V_{T}^{\Delta} \\
& c \longmapsto v \quad w \longmapsto c^{\Delta}(w),
\end{aligned}
$$

where $c^{\Delta}$ is an approximation of the non-local velocity. For $c \in V_{T}^{\Delta}, v=G^{\Delta}(c)$ is defined by

$$
v_{i}^{0}=u^{0}\left(x_{i}\right), \quad v_{i}^{n+1}=v_{i}^{n}+\Delta t c_{i}^{n} \times\left\{\begin{array}{cc}
D_{x}^{+} v_{i}^{n} & \text { if } c_{i}^{n} \geq 0, \\
D_{x}^{-} v_{i}^{n} & \text { if } c_{i}^{n}<0 .
\end{array}\right.
$$

We are looking for a solution to (52). Our goal is to give an abstract error estimate between the continuous solution $u$ and the discrete solution $v$. To this end, we need to introduce a long series of assumptions. Our error estimate will be given in Theorem 7.1. We make the following assumptions.

(A1) CFL condition.

$$
\Delta t \leq \frac{\Delta x}{\sup _{i \in \mathbb{Z}}\left|c_{i}\left(v^{n}\right)\right|} .
$$

(A2) $(u)^{\Delta} \in U_{T}^{\Delta}$, where $(u)^{\Delta}$ is the restriction of the continuous solution $u$ of (51) to $I_{d}^{T}$.

(A3) $(c)^{\Delta} \in V_{T}^{\Delta}$, where $(c)^{\Delta}$ is the restriction of the non-local velocity $c[u]$ to $I_{d}^{T}$.

(A4) $U_{T}^{\Delta}$ and $V_{T}^{\Delta}$ are equi-Lipschitz and equibounded, respectively, in the sense that there is a constant $K$ such that, for every mesh $\Delta$,

$$
\left|D_{x}^{+} w\right| \leq K,|c| \leq K, \text { for every } w \in U_{T}^{\Delta}, c \in V_{T}^{\Delta} .
$$

$$
G^{\Delta}\left(V_{T}^{\Delta}\right) \subset U_{T}^{\Delta} \quad \text { for every } T .
$$

(A6) The discrete velocity $c^{\Delta}$ is stationary. That is, there is a map $\bar{c}^{\Delta}$ such that

$$
\bar{c}^{\Delta}\left(w\left(\cdot, t_{n}\right)\right)=c^{\Delta}(w)\left(\cdot, t_{n}\right)
$$

$$
c^{\Delta}\left(U_{T}^{\Delta}\right) \subset V_{T}^{\Delta} \text { for every } T .
$$

(A8) Stability of the operator $G^{\Delta}$ (see Proposition 5.3 ).

There is a constant $K>0$ such that for every mesh $\Delta$ satisfying the CFL condition (A1), for every $T$ and every $c_{1}, c_{2} \in V_{T}^{\Delta}$,

$$
\sup _{I_{d}^{T}}\left|G^{\Delta}\left(c_{2}\right)-G^{\Delta}\left(c_{1}\right)\right| \leq K T \sup _{I_{d}^{T}}\left|c_{2}-c_{1}\right| .
$$

(A9) Consistency of the discrete velocity $c^{\Delta}$.

There is a constant $K>0$ such that for every mesh $\Delta$ and every $T$,

$$
\sup _{I_{d}^{T}}\left|c[u]-c^{\Delta}\left(u^{\Delta}\right)\right| \leq K \Delta x,
$$

where $u$ is the solution of (51) and $u^{\Delta}=(u)^{\Delta}$ is the restriction of $u$ to $I_{d}^{T}$. 
(A10) Stability of the discrete velocity $c^{\Delta}$.

There is a constant $K>0$ such that for every mesh $\Delta$, for every $T \leq \bar{T}$, and every $w_{1}, w_{2} \in U_{T}^{\Delta}$,

$$
\sup _{I_{d}^{T}}\left|c^{\Delta}\left(w_{1}\right)-c^{\Delta}\left(w_{2}\right)\right| \leq K\left(\sup _{I_{d}^{T}}\left|w_{2}-w_{1}\right|+\Delta x\right) .
$$

We have the following abstract error estimate (see [3, 2]).

Theorem 7.1 (An abstract error estimate for a short time). Let us consider $\bar{T}>0$ and $\Delta x+\Delta t \leq 1$. Let us assume that (A1)-(A10) hold for any $T \leq \bar{T}$ and that there exists a unique continuous solution $u$ of (51) on $[0, \bar{T}]$. There then exists a constant $K^{\prime}>0$, depending on $\left|c^{\text {ext }}\right|_{L^{\infty}(\mathbb{R})}, P,\left|c^{0}\right|_{L^{1}(\mathbb{R})}$, the bound constants of $D_{x}^{+} v_{i}^{n}, D_{x}^{-} v_{i}^{n}$ and $\left|\left(u^{0}\right)_{x}\right|_{L^{\infty}(\mathbb{R})}$, and there exists a constant $0<\bar{T}^{*} \leq \bar{T}$ with $\bar{T}^{*}$ only depending on $\bar{T}$ and $K^{\prime}$, such that for every $T \leq \bar{T}^{*}$ we have

$$
\sup _{I_{d}^{T}}|u-v| \leq K^{\prime} \sqrt{\Delta x} \quad \text { if } \quad \Delta x \leq \frac{\bar{T}^{*}}{K^{\prime}} .
$$

7.2. Application of the abstract error estimate: Proof of Theorem 1.5. We check successively assumptions (A1) to (A10).

(1) We assume the CFL condition (12) which implies (A1) and (47) because

$$
\begin{aligned}
\sup _{j \in \mathbb{Z}}\left|c_{j+1}^{n}-c_{j}^{n}\right| & \leq \sup _{j \in \mathbb{Z}}\left|c_{j+1}^{n}\right|+\sup _{j \in \mathbb{Z}}\left|c_{j}^{n}\right| \\
& \leq 2 \sup _{j \in \mathbb{Z}}\left|c_{j}^{n}\right| \\
& \leq 2|c|_{L^{\infty}(\mathbb{R} \times(0,+\infty))} \\
& \leq 2\left(\left|c^{\mathrm{ext}}\right|_{L^{\infty}(\mathbb{R})}+P\left|c^{0}\right|_{L^{1}(\mathbb{R})}\right),
\end{aligned}
$$

which will allow us to apply Lemma 5.2

Here we will apply Theorem 7.1 with $\bar{T}=T_{0}$ given in (32) and with $T_{1}=\bar{T}^{*}, C=K^{\prime}$ given by Theorem 7.1, We recall the following notations (see (28) and (31)):

$$
K=\left|c^{\text {ext }}\right|_{L^{\infty}(\mathbb{R})}+P\left|c^{0}\right|_{L^{1}(\mathbb{R})} \quad \text { and } \quad L=\left|c_{x}^{\text {ext }}\right|_{L^{\infty}(\mathbb{R})}+P\left|c_{x}^{0}\right|_{L^{1}(\mathbb{R})} .
$$

(2) By Proposition 2.2 we have $(u)^{\Delta} \in U_{T}^{\Delta}$, where $u$ is the solution of (511).

(3) It is clear that $(c)^{\Delta} \in V_{T}^{\Delta}$, where $c=c[u]$ given by (2) for the solution $u$ of (51).

(4) It is also clear that, by definition, the sets $U_{T}^{\Delta}$ and $V_{T}^{\Delta}$ are equi-Lipschitz and equi-bounded, respectively.

(5) We now check that $G^{\Delta}\left(V_{T}^{\Delta}\right) \subset U_{T}^{\Delta}$. Let $c \in V_{T}^{\Delta}$. By Lemma 5.1 we have

$$
\left|\frac{G^{\Delta}(c)_{i+1}^{n}-G^{\Delta}(c)_{i}^{n}}{\Delta x}\right|=\left|\frac{v_{i+1}^{n}-v_{i}^{n}}{\Delta x}\right| \leq B^{n} .
$$

Moreover,

$$
B^{n}=B^{n-1}\left(1+\Delta t \sup _{i \in \mathbb{Z}}\left|\frac{c_{i+1}^{n-1}-c_{i}^{n-1}}{\Delta x}\right|\right) \leq B^{n-1}(1+L \Delta t) \leq B^{n-1} e^{L \Delta t} .
$$


We deduce that $B^{n} \leq B^{0} e^{L n \Delta t} \leq B^{0} e^{L T}$. Therefore,

$$
\left|\frac{G^{\Delta}(c)_{i+1}^{n}-G^{\Delta}(c)_{i}^{n}}{\Delta x}\right| \leq B^{0} e^{L T} .
$$

Similarly, by Lemma 5.2, we have

$$
\frac{G^{\Delta}(c)_{i+1}^{n}-G^{\Delta}(c)_{i}^{n}}{\Delta x} \geq b^{0} e^{-L T} .
$$

Thus, $G^{\Delta}(c) \in U_{T}^{\Delta}$ for all $c \in V_{T}^{\Delta}$, and then $G^{\Delta}\left(V_{T}^{\Delta}\right) \subset U_{T}^{\Delta}$ for all $T$.

(6) We now consider the discrete non-local velocity given in (91), (10):

$$
c^{\mathrm{int}, \Delta}=c_{i}^{\mathrm{int}, n}=\sum_{l \in \mathbb{Z}} c_{i-l}^{0} E\left(v_{l}^{n}\right) \Delta x, \quad c_{i}^{0}=\frac{1}{\Delta x} \int_{I_{i}} c^{0}(x) d x
$$

with $I_{i}=\left(x_{i}-\frac{\Delta x}{2}, x_{i}+\frac{\Delta x}{2}\right)$. It is clearly stationary. We recall from [3] that $c_{i}^{\text {int, } n}$ can be written as the continuous convolution

$$
c_{i}^{\mathrm{int}, n}=c^{0} \star E\left(v_{\#}\right)\left(x_{i}\right),
$$

where $v_{\#}$ is the piecewise constant lifting of $v$

$$
v_{\#}=\sum_{i} v_{i} 1_{I_{i}}
$$

Obviously $c_{i}^{\text {ext }}$ is stationary. Therefore $c^{\Delta}$ is stationary.

(7) We now check that $c^{\Delta}\left(U_{T}^{\Delta}\right) \subset V_{T}^{\Delta}$. Indeed, for all $v \in U_{T}^{\Delta}$, we have

$$
\left|c^{\Delta}(v)\right| \leq K, \quad\left|D^{+} c^{\Delta}(v)\right| \leq\left|\frac{\partial}{\partial x}\left(c^{0} \star E\left(v_{\#}\right)\right)\right|_{L^{\infty}(\mathbb{R})} \leq L .
$$

Therefore $c^{\Delta}\left(U_{T}^{\Delta}\right) \subset V_{T}^{\Delta}$.

(8) The assumption (A7) holds by Proposition 5.3 .

(9) Consistency of the discrete velocity $c^{\text {int, } \Delta}$. We estimate:

$$
\begin{aligned}
& \sup _{i \in \mathbb{Z}}\left|c_{i}^{\text {int, }, \Delta}\left(u^{\Delta}\right)\left(\cdot, t_{n}\right)-c[u]\left(x_{i}, t_{n}\right)\right| \\
\leq & \sup _{x \in \mathbb{R}}\left|c^{0} \star E\left(u_{\#}^{\Delta}\right)\left(\cdot, t_{n}\right)-c^{0} \star E(u)\left(\cdot, t_{n}\right)\right| \\
\leq & \left|c^{0}\right|_{L_{\text {int }}^{\infty}(\mathbb{R})}\left|E\left(u_{\#}\right)\left(\cdot, t_{n}\right)-E(u)\left(\cdot, t_{n}\right)\right|_{L_{\text {unif }}^{1}(\mathbb{R})} \\
\leq & \frac{4 P}{b}\left|c^{0}\right|_{L_{\text {int }}^{\infty}(\mathbb{R})}\left|u_{\#}\left(\cdot, t_{n}\right)-u\left(\cdot, t_{n}\right)\right|_{L^{\infty}(\mathbb{R})} .
\end{aligned}
$$

Then $c^{\text {int, } \Delta}$ is consistent.

(10) Stability of the discrete velocity $c^{\text {int, } \Delta}$. We estimate

$$
\begin{aligned}
\left|c_{i}^{\mathrm{int}, \Delta}\left(w^{1}\right)-c_{i}^{\mathrm{int}, \Delta}\left(w^{2}\right)\right| & =\left|c^{0} \star E\left(w_{\#}^{1}\right)\left(x_{i}\right)-c^{0} \star E\left(w_{\#}^{2}\right)\left(x_{i}\right)\right| \\
& \leq\left|c^{0}\right|_{L_{\mathrm{int}}^{\infty}(\mathbb{R})}\left|E\left(w_{\#}^{1}\right)-E\left(w_{\#}^{2}\right)\right|_{L_{\mathrm{unif}}^{1}(\mathbb{R})} \\
& \leq 4 P\left|c^{0}\right|_{L_{\mathrm{int}}^{\infty}(\mathbb{R})}\left(\frac{1}{b} \sup _{i \in \mathbb{Z}}\left|w_{i}^{1}-w_{i}^{2}\right|+\Delta x\right),
\end{aligned}
$$

where we have used in the last time Proposition 6.1

Finally, we apply Theorem 7.1 and we obtain Theorem 1.5 


\section{EXAMPLE OF A SIMULATiON}

In this section, we provide some numerical simulations showing the behavior of the solution and the dislocations dynamics through obstacles.

We start by an initial data $u^{0}(x)=2 x$. The velocity is chosen as

$$
c[u](x, t)=A+B \sin (2 k \pi x)+c^{0} \star E(u(\cdot, t))(x),
$$

with $A=1.2, B=1$, the number of obstacles is $k=2$, the kernel $c^{0}$ is the one of Peierls Nabarro given by (44) with $\frac{\mu b^{2}}{2 \pi(1-\nu)}=1$ and $\zeta=0.1$. We choose $\Delta x=0.0099$ and $\Delta t=0.00263$. Numerically, we work on the interval for $x \in\left[-\frac{1}{2}, \frac{1}{2}\right]$.

In Figure 3, we represent the solution $u(x, t)$ as a function of $x \in\left[-\frac{1}{2}, \frac{1}{2}\right]$ for different values of $t=0,2,4,8$. In this figure we see that the gradient of the solution remains numerically in time bounded from above and from below, even if the lower bound of the gradient is very small. In Figure 4 we represent the trajectories of the dislocations $x(t)$ (here there are two dislocations) with the time on the vertical axis and the space on the horizontal one. We recall that the positions of dislocations correspond to the jumps of the floor part of the solution. In Figure 4 we see that the dislocations slow down on the obstacles. Finally, we remark numerically on Figure 3 that the gradient of the solution is far from zero in the regions where we take the floor part of the solution, which is a good behavior for this simulation. We can even say that we can localize the dislocations by the strong variations of the solution.

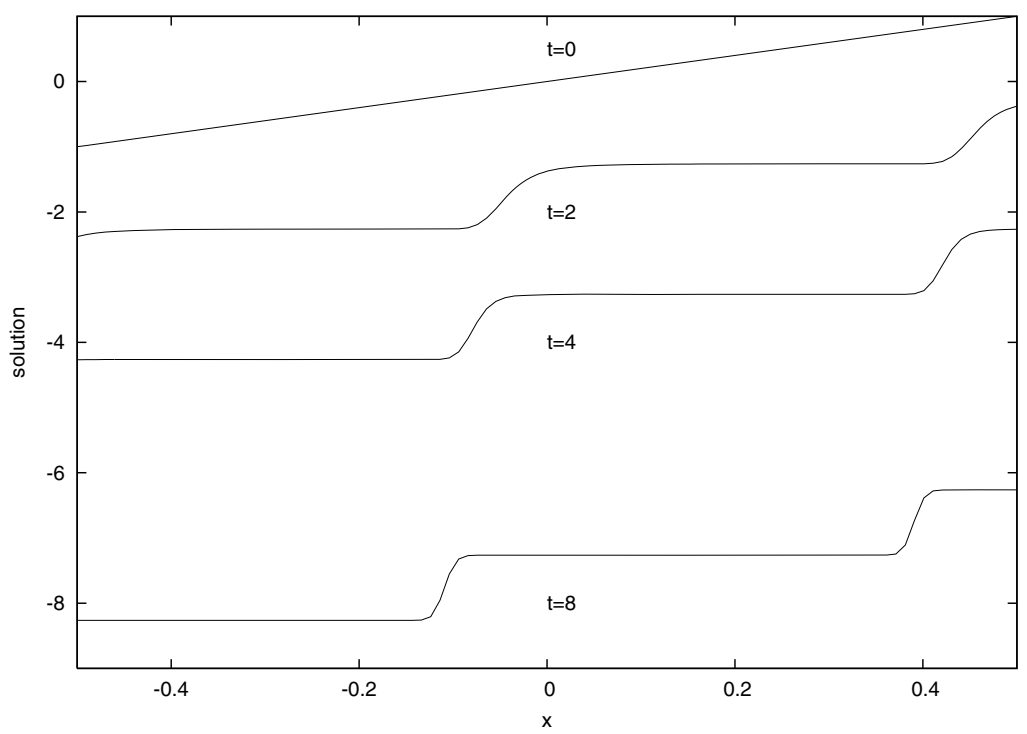

Figure 3. Behavior of the solution in time 


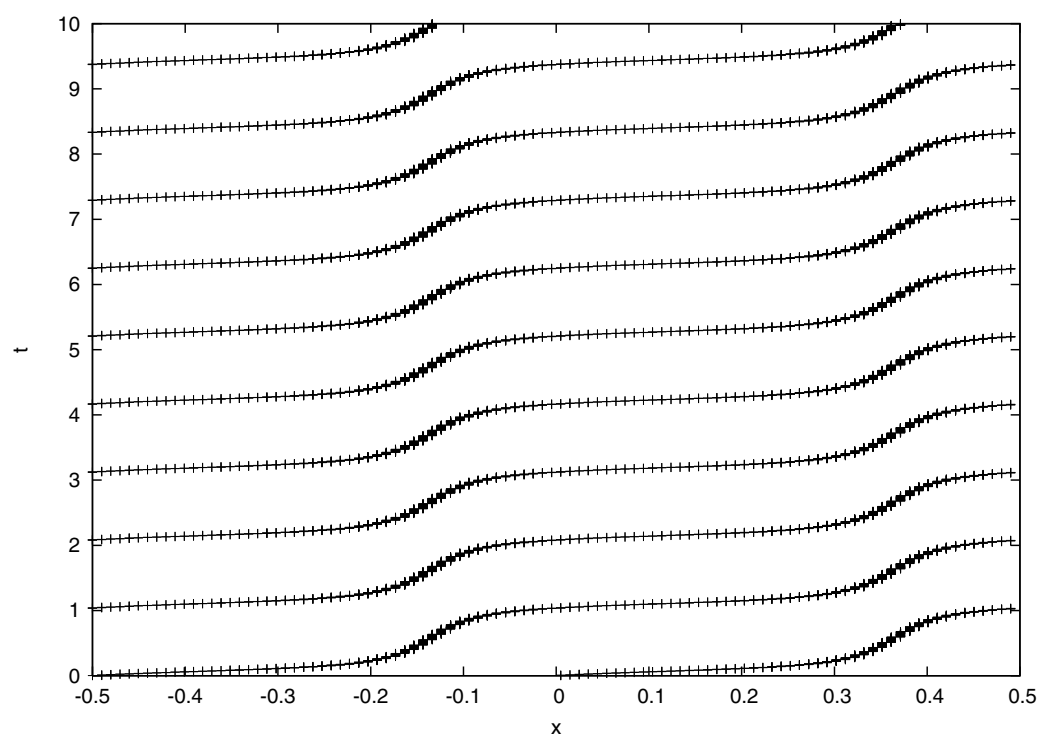

FIgURE 4. Dislocations dynamics through obstacles

\section{ACKNowledgements}

The authors would like to thank O. Alvarez, E. Carlini, A. Finel, P. Hoch, C. Imbert and Y. Le Bouar for fruitful discussions. We also thank A. El-Hajj and N. Forcadel for their comments. This work was supported by the contract JC 1025 called "ACI jeunes chercheuses et jeunes chercheurs" of the French Ministry of Research (2003-2006).

\section{REFERENCES}

[1] O. Alvarez, P. Cardaliaguet and R. Monneau, Existence and uniqueness for dislocation dynamics with nonnegative velocity, Interfaces and Free Boundaries, 7 (4), 415-434, (2005). MR2191694 (2006i:35023)

[2] O. Alvarez, E. Carlini, R. Monneau and E. Rouy, Convergence of a first order scheme for a non local eikonal equation, Appl. Numer. Math. 56 (9), 1136-1146, (2006). MR2244967 (2007d:65072)

[3] O. Alvarez, E. Carlini, R. Monneau and E. Rouy, A convergent scheme for a non local Hamilton Jacobi equation modelling dislocation dynamics, Numer. Math. 104 (4), 413-444, (2006). MR2249672 (2008f:74041)

[4] O. Alvarez, P. Hoch, Y. Le Bouar and R. Monneau, Résolution en temps court d'une équation de Hamilton-Jacobi non locale décrivant la dynamique d'une dislocation, C. R. Acad. Sci. Paris, Ser. I, 338, 679-684, (2004). MR2065373(2005b:35021)

[5] O. Alvarez, P. Hoch, Y. Le Bouar and R. Monneau, Dislocation dynamics: short time existence and uniqueness of the solution, Arch. Ration. Mech. Anal., 181 (3), 449-504, (2006). MR2231781 (2007d:74019)

[6] M. Bardi and I. Capuzzo-Dolcetta, Optimal Control and Viscosity Solutions of HamiltonJacobi-Bellman Equations, Birkhäuser, Boston, (1997). MR1484411(99e:49001)

[7] G. Barles, Solutions de Viscosité des Equations de Hamilton-Jacobi, Springer-Verlag, Berlin, (1994). MR 1613876(2000b:49054)

[8] G. Barles, P. Cardaliaguet, O. Ley and R. Monneau, General results for dislocation type equation, SIAM J. Math. Anal., 40 (1), 44-69, (2008). MR2403312(2009d:49049) 
[9] G. Barles and O. Ley, Nonlocal first-order Hamilton-Jacobi equations modelling dislocations dynamics, Comm. Partial Differential Equations, 31 (7-9), 1191-1208, (2006). MR.2254611 (2007h:35017)

[10] M.G. Crandall, H. Ishii and P.-L. Lions, User's guide to viscosity solutions of second order partial differential equations, Bull. Amer. Math. Soc. (N.S.), 27, 1-67, (1992). MR.1118699 (92j:35050)

[11] M.G. Crandall and P.-L. Lions, Conditions d'unicité pour les solutions généralisées des équations de Hamilton-Jacobi du premier ordre, C. R. Acad. Sci. Paris Ser. I Math., 292, 183-186, (1981). MR610314 (82c:49020)

[12] M.G. Crandall and P.-L. Lions, On existence and uniqueness of solutions of Hamilton-Jacobi equations, Nonlinear Anal. 10, 353-370, (1986). MR836671 (87f:35052)

[13] M.G. Crandall and P.-L. Lions, Two Approximations of Solutions of Hamilton-Jacobi Equations, Math. Comp., 167, 1-19, (1984). MR744921 (86j:65121)

[14] F. Da Lio, N. Forcadel and R. Monneau, Convergence of a non-local eikonal equation to anisotropic mean curvature motion. Application to dislocations dynamics, J. Eur. Math. Soc. (JEMS), 10 (4), 1061-1104, (2008). MR2443929

[15] N. Forcadel, Dislocations dynamics with a mean curvature term: short time existence and uniqueness, Differential and Integral Equations, 21 (3-4), 285-304, (2008). MR2484010

[16] A. Ghorbel, P. Hoch and R. Monneau, A numerical study for the homogenisation of onedimensional models describing the motion of dislocations, Int. J. of Computing Science and Mathematics, Vol. 2, Nos. 1/2, 28-52, (2008).

[17] A. Ghorbel and R. Monneau, Equation d'Hamilton-Jacobi non-locale modélisant la dynamique des dislocations, Proceedings of the 2nd TAM-TAM (Trends in Applied Mathematics in Tunisia, Algeria, Morocco), 322-328, (2005).

[18] J.P. Hirth and J. Lothe, Theory of dislocations, Second Edition. Malabar, Florida: Krieger, (1992).

[19] S. Osher, J.A. Sethian, Fronts Propagating with Curvature-Dependent Speed: Algorithms Based on Hamilton-Jacobi Formulations, J. Comp. Physics, 79, 12-49, (1988). MR965860 (89h:80012)

[20] D. Rodney, Y. Le Bouar and A. Finel, Phase field methods and dislocations, Acta Materialia $51,17-30,(2003)$

Cermics, École Nationale des Ponts et Chaussées, 6 et 8 avenue Blaise Pascal, Cité Descartes, Champs-sur-Marne, 77455 Marne-la-Vallée Cédex 2, France

E-mail address: ghorbel@cermics.enpc.fr

Cermics, École Nationale des Ponts et Chaussées, 6 et 8 avenue Blaise Pascal, Cité Descartes, Champs-sur-Marne, 77455 Marne-la-Vallée Cédex 2, France

E-mail address: monneau@cermics.enpc.fr 